\title{
Variable-Length Input Huffman Coding for System-on-a-Chip Test*
}

\author{
Paul. T. Gonciari, Bashir M. Al-Hashimi, and Nicola Nicolici
}

Paper no. 556

Accepted for publication as a Transaction Brief Paper

Submitted: July 2002, Revised: October 2002, Final manuscript: January 2003

\section{Paul T. Gonciari and Bashir M. Al-Hashimi}

Electronic Systems Design Group

Department of Electronics and Computer Science

University of Southampton

Southampton SO17 1BJ, U.K.

Tel: +44-23-8059-3119/+44-23-8059-3249 Fax: +44-23-8059-2901

Email: \{p.gonciari@zepler.org,bmah@ecs.soton.ac.uk\}

\section{Nicola Nicolici}

Computer-Aided Design and Test Research Group

Department of Electrical and Computer Engineering

McMaster University

1280 Main St. W., Hamilton, ON L8S 4K1, Canada

Tel: +1-905-525-9140 ext. 27598 Fax: +1-905-521-2922

Email:nicola@ece.mcmaster.ca

\footnotetext{
${ }^{*}$ A preliminary version of this paper was published in Design Automation and Test in Europe (DATE) 2002, pp. 604-611
} 


\title{
Variable-Length Input Huffman Coding for System-on-a-Chip Test
}

\begin{abstract}
This paper presents a new compression method for embedded core-based system-on-a-chip test. In addition to the new compression method, this paper analyzes the three test data compression environment (TDCE) parameters: compression ratio, area overhead and test application time, and explains the impact of the factors which influence these three parameters. The proposed method is based on a new Variable-length Input Huffman Coding scheme, which proves to be the key element that determines all the factors that influence the TDCE parameters. Extensive experimental comparisons show that, when compared to three previous approaches [1-3], which reduce some test data compression environment's parameters at the expense of the others, the proposed method is capable of improving on all the three TDCE parameters simultaneously.
\end{abstract}




\section{Introduction}

Due to the increased complexity of systems-on-a-chip (SOC), testing is an important factor that drives time-to-market and the cost of the design [4]. In addition to the standard design for test (DFT) issues for SOCs [4], a new emerging problem is the cost of the automatic test equipment (ATE) [5-7]. This is because the cost of ATE grows significantly with the increase in speed (i.e., operating frequency), channel capacity, and memory size [6]. In order to support the large volume of test data with limited channel capacity for future SOCs, the ATE requires modifications and additional expenses. Furthermore, the testing time is also dependent on the volume of test data, thus further increasing the manufacturing cost of the SOCs [7]. The above problems were previously addressed using (a) external-only approaches (no on-chip overhead is required) or $(b)$ internal-only approaches (no off-chip interaction is required). The external-only approaches $(a)$ include test data compaction $[8,9]$ and methods which reduce the amount of data transfered from the workstation to the ATE [10,11]. While these approaches effectively reduce the volume of test data, they do not reduce the ATE bandwidth requirements. The internalonly approaches $(b)$ are based on built-in self-test (BIST), i.e., pseudo-random and/or deterministic BIST. To increase the fault coverage of pseudo-random BIST intrusive techniques such as test point insertion are required [5]. Such techniques are not acceptable in a core-based SOC environment since they lead to a major core redesign effort. In addition, when intellectual property (IP) protected cores are targeted, intrusive techniques cannot be applied. To alleviate the above problems related to pseudorandom BIST, deterministic BIST [12-17] approaches have emerged. These however, may require large on-chip area in order to achieve high test quality. Therefore, test data compression, a test resource partitioning variant [6], arises as a possible solution to reducing the speed, the channel capacity and the memory requirements for ATEs. This solution does not introduce performance penalties and guarantees full reuse of the existing embedded cores as well as the ATE infrastructure, with modifications required during system test preparation (compression) and test application (decompression). Prior work with respect to test data compression is overviewed in the following section.

\subsection{Existing approaches to reduce volume of test data}

Test data compression is a test resource partitioning scheme whose main target is the reduction of volume of test data. By introducing on-chip decompression hardware it reduces the load on the ATE, and 
therefore it simplifies the ATE channel capacity and speed requirements. Test data compression methods can reduce the volume of test data applied to the core under test (CUT) or the volume of test responses sent to the ATE. Test response compression is carried out using signature analyzers [18], however, if diagnosis is necessary special schemes may be required $[19,20]$. In this paper, we will focus on the former due to the large volumes of test data required to test logic cores, assuming signature analyzers to compress the test output responses. Test data compression methods, which reduce the volume of test data applied to the CUT, can be classified into approaches $(i)$ which extend deterministic BIST [21-25], (ii) which exploit the spareness of care bits in the test set [26-28], and (iii) which exploit the regularities within the test set [1-3,29-33]. The first category [21-25] (i), enhances deterministic BIST approaches, thus alleviating some of their drawbacks (e.g., area overhead, test application time). However, these approaches may suffer from inefficient tester channel usage such as ATE inactivity periods which may increase cost [28]. The second category [26-28] (ii), by exploiting care bit spareness, can reduce the volume of test data when the percentage of "don't care” bits is high. However, it suffers from lockout situations [12], i.e., the inability of the on-chip decoder to generate a given test pattern, which can degrade test quality, increase test control complexity, as well as negatively influence test application time (TAT). These lock-out situations can increase significantly when the percentage of "don't care" bits in a test set decreases. Due to these issues the above approaches [26-28] are embedded into the ATPG procedures, which may be prohibited in the IP-based tool flow, where the system integrator cannot access the structural information of the embedded cores. The third category [1-3,29-33] (iii), by exploiting the regularities within the test set, does not require any ATPG re-runs. Since, ATPG procedures require fault simulation, which may be prohibited in IP environments, and long ATE inactivity periods can increase the cost, in this paper we will focus on the third category (iii) [1-3,29-33].

Methods which use test set regularities exploit different features of the test set, such as runs of '0's, and/or small differences between consecutive test vectors. These methods are based on data compression coding schemes (e.g., run-length, Huffman, Golomb [34]) [1-3, 29, 30] and custom coding schemes [3133]. The above coding schemes are implemented using on-chip hardware decoders or on-chip software decoders. With respect to on-chip hardware decoders, Iyengar et al. [29], proposed a new built-in scheme for testing sequential circuits based on statistical coding. Results indicate that the method is suitable for circuits with a relatively small number of primary inputs. To overcome this disadvantage a selective 
coding (SC) algorithm was proposed [1]. The method splits the test vectors into fixed-length block size patterns ${ }^{1}$ and applies Huffman coding to a carefully selected subset. In [30], test vector compression is performed using run-length coding. The method relies on the fact that successive test vectors in a test sequence often differ in only a small number of bits. Hence, the initial test set $T_{D}$ is transformed into $T_{d i f f}$, a difference vector sequence, in which each vector is computed as the difference between two consecutive vectors in the initial test set. Despite its performance, the method is based on variable-tofixed-length codes and, as demonstrated in [2], these are less effective than variable-to-variable-length codes. Chandra and Chakrabarty [2] introduced a compression method based on Golomb codes. Both methods, [2] and [30], use the difference vector sequence, hence, in order to generate the initial test set on-chip, a cyclical scan register (CSR) [2,30] architecture is required. In [3], a new method based on frequency-directed run-length (FDR) codes is proposed. The coding scheme exploits a particular pattern distribution, and by means of experimental results it is shown that the compression ratio can be improved. In [33], a method which exploits the correlation between two consecutive parallel loads into multiple scan chains has been proposed. Since the method uses multiple scan chains it reduces the testing time, however, the extra control signals may negatively influence the compression ratio and control complexity. In addition to the above approaches, which require an on-chip hardware decoder, methods which assume the existence of an embedded processor [31,32], hence an on-chip software decoder, were also proposed. The method in [31] is based on storing the differing bits between two test vectors, while the method in [32] uses regular geometric shapes formed only from '0's or '1's to compress the test data. Regardless of their benefits, in systems where embedded processors are not available or where embedded processors have access only to a small number of embedded cores, these on-chip software decoder based methods are not applicable. A particular advantage of methods which use data compression coding schemes is that they are capable of exploiting the ever increasing gap between the on-chip test frequency and the ATE operating frequency. While in the past this gap has been exploited at the cost of multiple ATE channels [35,36], hence increasing the bandwidth requirements, approaches which use data compression coding schemes can leverage the frequency ratio without the penalty of extra ATE channels. This is achieved by moving the serialization of test data from the spatial

\footnotetext{
${ }^{1}$ It should be noted that throughout the paper, a pattern refers to an input pattern used in the coding scheme and not to a test pattern. A coding scheme uses fixed-length patterns if all the input patterns have the same length, otherwise, it uses variable-length patterns.
} 
domain (multiple input channels from the ATE at a low frequency to single scan channel at a high frequency) to the temporal domain (single input channel from the ATE at a low frequency to single scan channel at a high frequency). Hence, data can be fed to the internal cores at their corresponding test frequency without any bandwidth penalty.

\subsection{Motivation and contributions}

Since so many compression methods have been proposed, an obvious question is why yet another approach is necessary? When analyzed with respect to the three test data compression environment parameters, which are compression ratio, area overhead and test application time, previous approaches trade-off some of the parameters against the others. Therefore, the motivation behind this work is to understand the factors which influence these parameters and to propose a new approach which, when compared to previous approaches, allows simultaneous improvement in compression ratio, area overhead and test application time. This will lead to reduced ATE memory requirements, lower chip area and shorter time spent on the tester, hence reduced test cost. The three main contributions of this paper are:

- The test data compression environment (TDCE) comprising the compression method and the onchip decoder is defined and analyzed with respect to the three TDCE parameters: compression ratio, area overhead and test application time. The interrelation between different factors which influence these parameters is discussed and an analysis of previous work with respect to the TDCE is also given. To the best of our knowledge, this is the first paper which explicitly illustrates the influences of the various factors on the two main components of TDCE (see Section 2);

- A new coding scheme, Variable-length Input Huffman Coding (VIHC) is proposed and it is shown that the recently proposed Golomb based method is a particular case of the proposed coding scheme, leading to constantly lower compression ratios. A new compression algorithm comprising a new reordering and a new mapping algorithm is also given;

- A new parallel on-chip decoder is proposed. The parallel on-chip decoder has lower area overhead and it reduces the testing time when compared to the previous approaches. The test application time is analyzed and it is shown that the derived approximation function is close to the experimental data. Extensive experimental results show that, when the proposed coding scheme and the new 
parallel on-chip decoder are combined, the three test data compression environment parameters can be simultaneously improved with respect to previous work.

The remainder of the paper is organized as follows. Section 2 introduces the test data compression environment and analyzes three previous approaches with respect to its parameters. Section 3 introduces the new Variable-length Input Huffman Coding scheme and illustrates the proposed compression algorithm. In Section 4 the proposed on-chip hardware decoder is introduced and the TAT analysis is given. Section 5 and 6 provide the experimental results and conclusions, respectively.

\section{Test data compression environment (TDCE)}

To better understand how the volume of test data, the area overhead and the testing time can be simultaneously reduced, this section introduces the test data compression environment and characterizes the TDCE with respect to the factors which influence it. Three previous approaches are also analyzed from the TDCE parameters standpoint. Testing in TDCE implies sending the compressed test data from the ATE to the on-chip decoder, decompressing the test data on-chip and sending the decompressed test data to the core under test (CUT). There are two main components in TDCE: the compression method, used to compress the test set off-chip, and the associated decompression method, based on an on-chip decoder, used to restore the initial test set on-chip. The on-chip decoder comprises two units: a unit to identify a compressed code and a unit to decompress it. If the two units can work independently (i.e., decompressing the current code and identifying a new code can be done simultaneously), then the decoder is called parallel. Otherwise, the decoder is referred to as serial.

\subsection{TDCE characterization}

Testing in TDCE is characterized by the following three parameters: (a) compression ratio which identifies the performance of the compression method, the memory and channel capacity requirements of the ATE; (b) area overhead imposed by the on-chip decoder (dedicated hardware or on-chip processor); and (c) test application time given by the time needed to transport and decode the compressed test set.

There are a number of factors which influence the above parameters:

- the mapping and reordering algorithm, which prepares the test set for compression by mapping the "don't cares" in the test set to '0's or '1's, and by reordering the test set; 


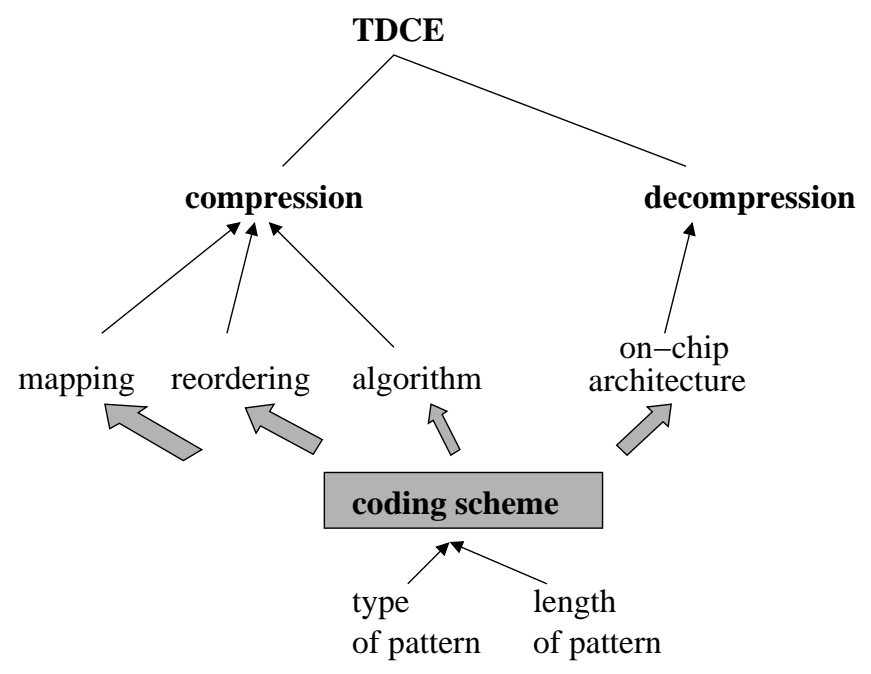

Figure 1. TDCE dependency map

- the compression algorithm, which based on a coding scheme compresses the initial test set;

- the type of input patterns used as input by the coding scheme, which can be of fixed or variable lengths;

- the length of the pattern which is the maximum allowed input pattern length used in the coding scheme;

- the type of the on-chip decoder, i.e., the on-chip decoder can be serial or parallel.

The relationship between these factors and the two components of the TDCE is illustrated in Figure 1. As it can be seen in the figure, the coding scheme not only determines the compression factors (i.e., mapping/reordering and compression algorithm), but it also influences the decompression factor (i.e., on-chip architecture). Hence, the coding scheme is a key element that determines the factors that influence the three TDCE parameters, which are discussed next.

(a) Compression ratio: Using patterns of various types and of various lengths, the compression algorithms exploit different features of the test set. Mapping and reordering the initial test set emphasizes these features. Therefore, the compression ratio is influenced firstly by the mapping and reordering algorithm, and then by the type of input patterns and the length of the pattern, and finally by the compression algorithm. 
(b) Area overhead: Area overhead is influenced firstly by the nature of the decoder, and then by the type of the input pattern and the length of the pattern. If the decoder is serial then the synchronization between the two units (code identification and decompression) is already at hand. However, if the decoder is parallel, then the two units have to synchronize, which can lead to increased control complexity and consequently to higher area overhead. Depending on the type of the input pattern different types of logic are required to generate the pattern on-chip. For example, if the coding scheme uses fixed-length input patterns, then a shift register is required to generate the patterns, however, if variable-length input patterns (runs of '0's for example) are used, then counters can be employed to generate the patterns. Since the length of the pattern impacts the size of the decoding logic, it also influences the area overhead.

(c) Test application time (TAT): TAT is firstly influenced by the compression ratio, and then by the type of the on-chip decoder, and the length of the pattern. To illustrate the factors that influence TAT, consider the ATE operating frequency as the reference frequency. Minimum TAT $\left(\min _{T A T}\right)$ is given by the size of the compressed test set in ATE clock cycles. However, this TAT can be obtained only when the on-chip decoder can always process the currently compressed bit before the next one is sent by the ATE. In order to do so, the relation between the frequency at which the on-chip decoder works and the ATE operating frequency has to meet certain conditions. The frequency ratio is the ratio between the onchip test frequency $\left(f_{\text {chip }}\right)$ and the ATE operating frequency $\left(f_{\text {ate }}\right)$. Consider that the optimum frequency ratio is the frequency ratio for which $\min _{T A T}$ is obtained. Since the $\min _{T A T}$ is given by the size of the compressed test set, increasing the compression ratio would imply further reduction in TAT. However, this reduction happens only if the optimum frequency condition is met. Since real environments cannot always satisfy the optimum frequency ratio condition, then a natural question is what happens if this condition is not met? TAT in these cases is dependent on the type of on-chip decoder. If the on-chip decoder has a serial nature $[2,3]$, then the TAT is heavily influenced by changes in the frequency ratio. However, if the decoder has a parallel nature [1], the influences are rather minor. The length of the pattern determines the pattern distribution and at the same time, the number of clock cycles the on-chip decoder requires to generate a pattern. Therefore, the length of the pattern determines the optimum frequency ratio and, hence, it also influences the TAT. 


\subsection{Analysis of previous approaches in TDCE}

In this section three representative previous approaches [1-3] are analyzed from the TDCE parameters standpoint and it is shown that they have improved some of the parameters at the expense of the others.

(i) Selective coding (SC) [1]: This method splits the test vectors into fixed-length input patterns of size $b$ (block size), and applies Huffman coding to a carefully selected number of patterns while the rest of the patterns are prefixed. The SC decoder has a parallel nature. Although, due to this parallelism, the on-chip decoder yields good TAT, the use of fixed-length input patterns and prefixed codes requires shift registers of length $b$, which lead to large area overhead. In addition, fixed-length input patterns restrain the method from exploiting '0'-mapped test sets. Hence, special pre-processing algorithms [37,38] have been proposed to increase the compression attainable with SC. However, these algorithms, which target the SC fixed-length input pattern principle, further increase the computational complexity of this method. It is also interesting to note that using SC with $T_{\text {diff }}$ allows good compression when the block size is increased. Hence, due to a fixed-length input pattern the method is restrained from exploiting '0'-mapped test sets, and the main drawback of this approach is its large area overhead.

(ii) Golomb coding [2]: This method assigns a variable-length Golomb code, of group size $m_{g}$, to a run of '0's. Golomb codes are composed from two parts, a prefix and a tail, and the tail has the length given by $\log _{2} m_{g}$. Using runs of '0's as input patterns, the method has two advantages: firstly the decoder uses counters of length $\log _{2} m_{g}$ instead of shift registers, thus leading to low area overhead; and secondly it exploits the ' 0 '-mapped test sets improving the compression ratio. Golomb codes are optimum only for a particular pattern distribution, hence the coding scheme yields best compression only in particular cases. Furthermore, since the method in [2] uses a serial on-chip decoder, the TAT is heavily influenced by changes in the frequency ratio. Therefore, the method's main drawback is its large TAT.

(iii) Frequency-directed run-length (FDR) coding [3]: The FDR code is composed from two parts, a prefix and a tail, where both parts have the same length. In order to decompress a FDR code, the on-chip decoder has to identify the two parts. Because the code is not dependent on a group size, as the Golomb code, the decoder has to detect the length of the prefix in order to decode the tail. In order to do so, the FDR code requires a more complicated decoder with fixed area overhead. The code being 
developed to exploit particular pattern distributions common to most test sets yields good compression ratios. However, despite the good compression ratios, having a serial on-chip decoder, large TAT is the main disadvantage of the FDR method.

As illustrated above, current approaches for test data compression efficiently address some of the test parameters at the expense of the others. Therefore, this paper proposes a new coding scheme and a new on-chip decoder, which when combined allow simultaneous improvement in all the three TDCE's parameters, when compared to previous work.

\section{Compression}

As illustrated in Section 2.1, the coding scheme is the key component in the TDCE. This section introduces a new Variable-length Input Huffman Coding scheme (Section 3.1) and a new compression algorithm which combines the new coding scheme with a mapping and reordering algorithm (Section 3.2).

\subsection{New Variable-length Input Huffman Coding (VIHC) scheme}

The proposed coding scheme is based on Huffman coding. Huffman coding is a statistical datacoding method that reduces the average code length used to represent the unique patterns of a set [34]. It is important to note that the previous approach [1] which uses Huffman coding in test data compression employs only patterns of fixed-length as input to the Huffman coding algorithm. As outlined in the previous section fixed-length input patterns restrict exploitation of the test set features for compression. This problem is overcome by the coding scheme proposed in this section which uses patterns of variable-length as input to the Huffman algorithm, allowing an efficient exploitation of test sets which exhibit long runs of '0's (Section 3). This fundamental distinction does not influence only the compression, but it also provides the justification for employing a parallel decoder using counters (Section 4.1). This will lead not only to significantly lower area overhead, but it will also facilitate TAT reduction when the compression ratio and frequency ratio are increased (see Section 4.2 and 5). The following two examples, illustrate the proposed coding scheme and highlight some interesting properties.

Example 1 Figure 2 illustrates the proposed coding scheme for a group size of $4\left(m_{h}=4\right)$. The group size represents the maximum acceptable number of '0's contained in a run of '0's. Using the group size, the initial test vector $\left(t_{\text {init }}\right)$ is divided into runs of ' 0 's of length smaller than or equal to 4 , which are 


$$
\begin{aligned}
& \mathrm{m}_{\mathrm{h}}=4 \quad\left|\mathrm{t}_{\text {init }}\right|=26 \text { bits } \quad\left|\mathrm{t}_{\mathrm{cmp}}\right|=16 \text { bits }
\end{aligned}
$$

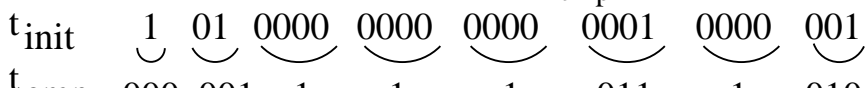

(a) Initial test vector

\begin{tabular}{l|c|c}
\multicolumn{1}{c|}{ Pattern } & Occurrence & Code \\
\hline $\mathrm{L}_{0}=1$ & 1 & 000 \\
$\mathrm{~L}_{1}=01$ & 1 & 001 \\
$\mathrm{~L}_{2}=001$ & 1 & 010 \\
$\mathrm{~L}_{3}=0001$ & 1 & 011 \\
$\mathrm{~L}_{4}=0000$ & 4 & 1
\end{tabular}

(b) Dictionary

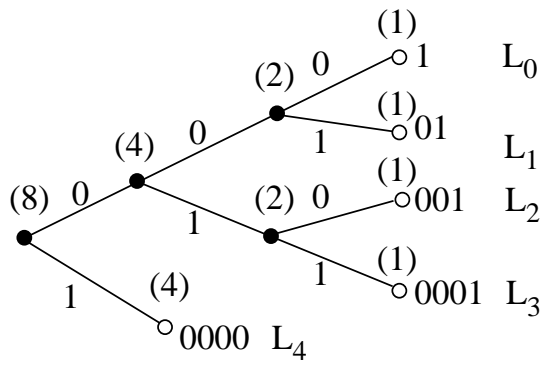

(c) Huffman tree

\begin{tabular}{r|l|l} 
Run of 0s & Golomb code & VIHC code \\
1 & 000 & 000 \\
01 & 001 & 001 \\
0000000000000001 & 1111011 & 1111011 \\
0000001 & 1010 & 1010
\end{tabular}

(d) Comparison with Golomb for $m_{h}=4$

\section{Figure 2. VIHC for $m_{h}=4$}

referred to as patterns (Figure 2(a)). These patterns are used as input to the Huffman coding scheme, where for each pattern the number of occurrences is determined. Having the patterns and the number of occurrences, the first two columns of the dictionary are filled (see Figure 2(b)). Using these two columns, the Huffman tree ${ }^{2}$ is built as explained next (see Figure 2(c)). First, the patterns are ordered in the descending order of their occurrences. Then, the two patterns with the lowest number of occurrences ( $L_{0}$ and $\left.L_{1}\right)$ are combined into a new node $\left(L_{0}, L_{1}\right)$ with the number of occurrences given by the sum of the two patterns' occurrences. This step is repeated until all the nodes are merged into one single node. Initially, patterns $L_{2}$ and $L_{3}$ are merged into $\left(L_{2}, L_{3}\right)$, then nodes $\left(L_{0}, L_{1}\right)$ and $\left(L_{2}, L_{3}\right)$ are merged together, and finally, node $L_{4}$ is merged with node $\left(\left(L_{0}, L_{1}\right),\left(L_{2}, L_{3}\right)\right)$. This process is illustrated in Figure 2(c), where the occurrences of the patterns are also given between brackets. After the tree is

\footnotetext{
${ }^{2}$ For a detailed description of the Huffman algorithm, the reader is referred to [34]
} 
created, the Huffman code is obtained by assigning binary '0's and '1's to each segment starting from the nodes of the Huffman tree (Figure 2(c)). The codes are illustrated in the third column in Figure 2(b). Using the obtained Huffman code, the initial test vector $t_{\text {init }}$ is compressed into $t_{c m p}$. It should be noted that ordering the patterns in the ascending or descending order of their occurrences does not make any difference to the Huffman tree building algorithm. It is important, however, to always combine the patterns with the smallest number of occurrences.

One interesting property, which can be easily observed when the proposed VIHC scheme and the Golomb coding scheme [2] are compared, is that Golomb codes represent a particular case of VIHCs. This is illustrated in Figure 2(d), where the codes obtained for the runs of ' 0 's from $t_{\text {init }}$ in Figure 2(a), are the same for both coding schemes. Because of this particularity, the compression ratios of the Golomb coding scheme will be upper bounded by the compression ratios of the VIHC coding scheme for a given group size. This is illustrated in the following example.

Example 2 Consider the scenario illustrated in Figure 3, where a simple change in the $t_{\text {init }}$ from Figure 2(a) is performed: the 24th bit is set from 0 to 1 . The dictionary and the Huffman tree for this new scenario are shown in Figure 3(b) and 3(c) respectively. The Golomb and the VIHC codes for this case are given in Figure 3(d). When $t_{\text {init }}$ is encoded using Golomb coding, the encoded test vector is 19 bits long $\left(t_{c m p}^{G}\right)$. If the test set is encoded using the proposed VIHC scheme, then the encoded test set is 17 bits long $\left(t_{c m p}^{V}\right)$, which shows the reduction in volume of test data.

In the following we formalize the proposed coding scheme and the properties illustrated using the previous examples. Let $m_{h}$ be the group size, and $L=\left\{L_{0}, \ldots, L_{m_{h}}\right\}$ be a set of unique patterns obtained after the test set was divided into runs of ' 0 's of maximum length $m_{h}$. For the computed set of patterns $L$, the set of number of occurrences $P=\left\{n_{0}, \ldots, n_{m_{h}}\right\}$ is determined. $n=\sum_{i=0}^{m_{h}}\left|L_{i}\right| * n_{i}$ is the size of the test set, where $\left|L_{i}\right|$ denotes the length of pattern $L_{i}$. The Huffman codes are obtained using $L$ and $P$. For a pattern $L_{i}$ the Huffman code is denoted by $c_{i}$, and the length of the code is given by $w_{i}$. The minimum codeword length $\min \left\{w_{i}\right\}$ is referred to as $w_{\min }$. The size of the compressed test set using a group size of $m_{h}$ is denoted by $H\left(m_{h}\right)=\sum_{i=0}^{m_{h}} n_{i} * w_{i}$. It should be noted that the terms "Huffman code" and "codeword" will be used interchangeably and the term "group size" is preferred to "block size". It is interesting to note that there are at most $m_{h}+1$ patterns and thus $m_{h}+1$ leafs in the Huffman tree. 


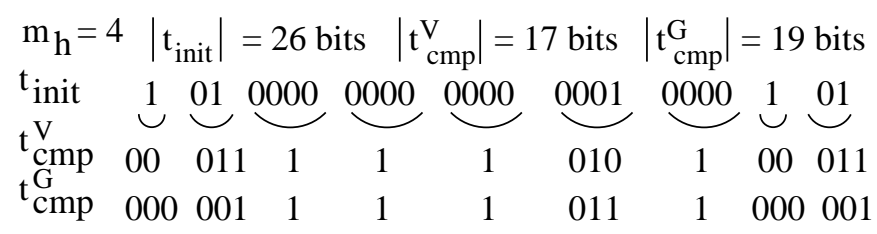

(a) Test vectors

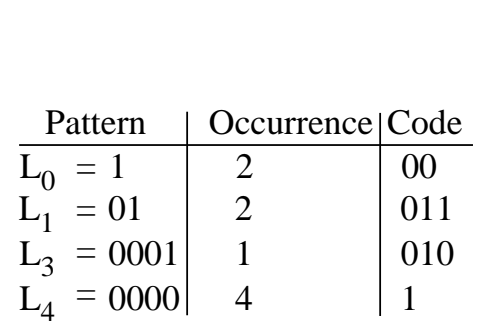

(b) Dictionary

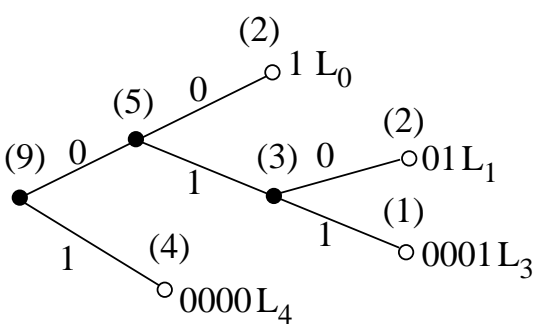

(c) Huffman tree

\begin{tabular}{r|l|l} 
Run of 0s & Golomb code & VIHC code \\
1 & 000 & 00 \\
01 & 001 & 011 \\
0000000000000001 & 111011 & 111010 \\
00001 & 1000 & 100
\end{tabular}

(d) Comparison with Golomb for $m_{h}=4$

Figure 3. VIHC, the general case

There are $m_{h}$ patterns formed from runs of ' 0 's ending in $1\left(L_{0} \ldots L_{m_{h}-1}\right)$ and one pattern formed from '0's only $\left(L_{m_{h}}\right)$ as illustrated in Figure 2(b) for $m_{h}=4$.

We observed in Example 1 that Golomb coding is a particular case of VIHC. Hence, for a given group size and for a particular pattern distribution the VIHC will reduce to the Golomb code. Consider the following pattern distribution: $(a) n_{m_{h}} \geq n_{i_{0}}+n_{i_{1}}+\ldots+n_{\frac{i m_{h}}{2}-1}, \forall$ distinct $i_{0} \ldots i \frac{m_{h}}{2}-1<m_{h}$ and (b) $n_{k} \geq n_{i}+n_{j}, \forall i, j, k<m_{h}, i \neq j \neq k$, where $m_{h}$ represents the group size and $n_{i}$ is the number of occurrences of pattern $L_{i}$. Based on the above conditions, the Huffman tree illustrated in Figure 4 is constructed. Condition $(a)$ guarantees that the pattern $L_{m_{h}}$ will be the left-most leaf in the tree (as shown in Figure 4). Condition (b) ensures that the Huffman tree construction algorithm will merge all the simple patterns first, while the merged patterns are processed afterwards, i.e., it will ensure that the simple patterns $\left(L_{0} \ldots L_{m_{h}-1}\right)$ are on the last level of the tree as illustrated in Figure 4 . It should be 


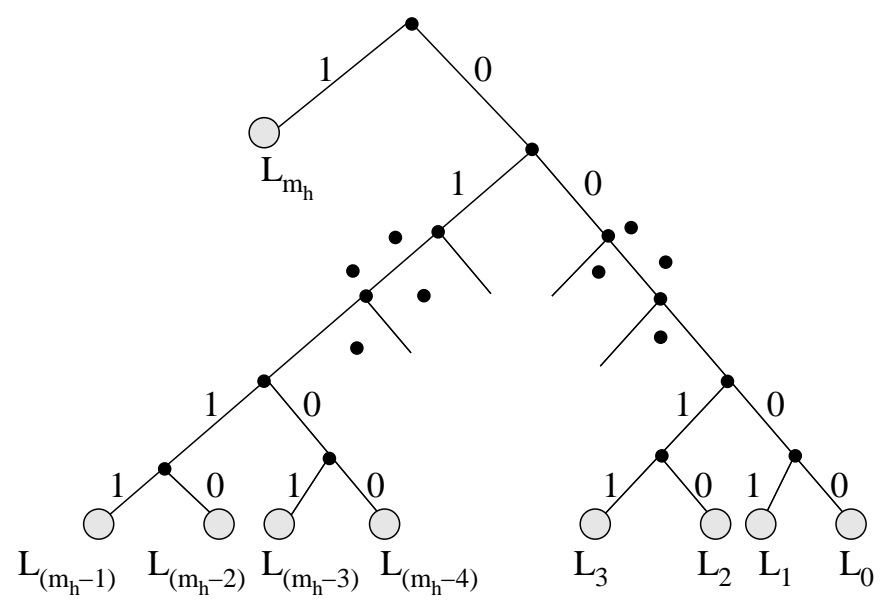

Figure 4. Huffman tree when the Golomb code and the VIHC are equal

noted that even if the patterns $L_{i}$, with $i<m_{h}$, appear in a different order than the one illustrated in the figure, then as long as the above conditions are met, swapping and rearranging the nodes in the Huffman tree will lead to the tree presented in Figure 4 with no penalties in compression ratio ${ }^{3}$ [34]. Using the convention that the left leaf of a node is assigned a ' 1 ' and the right one a ' 0 ', it is found that $c_{m_{h}}=1$ and $c_{i}=0$ followed by $i$ represented on $\log _{2} m_{h}$ bits. It is clear that if a run of '0's of length lower than $m_{h}$ is compressed, then the code is the same as Golomb's. If the length of run of '0's $(l)$ is greater than $m_{h}$, then the assigned Golomb code is given by $\underbrace{1 \ldots 1}_{\left\lfloor\frac{l}{m_{h}}\right\rfloor}$ Otail, where tail $=l-\left\lfloor\frac{l}{m_{h}}\right\rfloor$ represented on $\log _{2} m_{h}$ bits. The same representation is obtained if the code is derived using the proposed VIHC method. Since, the proposed method can produce other codes as well, depending on the pattern distribution for a given group size, the Golomb code is a particular case of the proposed coding method.

It is very interesting to note that the above observation ensures that the VIHC decoder proposed in Section 4.1 can be used to decompress a Golomb compressed test set. Furthermore, if the VIHC coding obtains the same code as Golomb, the minimum code length is 1 , or $w_{\min }=1$. This derives from the previous observation and can be easily seen in Figures 2(c) and 4. Moreover, as illustrated in Figure 3, Golomb coding leads to smaller compression ratios when compared to the proposed VIHC scheme. This is formalized in the following theorem.

\footnotetext{
${ }^{3}$ It should be noted that when $n_{k}=n_{i}+n_{j}$ in condition $(b)$, the Huffman algorithm may construct a tree different to the one shown in Figure 4. However, by rearranging the leafs, the tree in Figure 4 can be obtained without any penalties in compression ratio [34]
} 
Theorem 1 For a given group size, the compression obtained by the Golomb coding is lower than or equal to the compression obtained by VIHC.

Proof: The above can be easily shown by using the fact that the Huffman code is an optimal code ([34, Theorem 5.8.1]), i.e., any other code will have the expected length greater than the expected length of the Huffman code. Since the Golomb code is a particular case of VIHC (the VIHC in this particular case is referred to as $V I H C_{G}$ ), it is optimal only for a particular pattern distribution. For other pattern distributions the $\mathrm{VIHC}_{G}$ code is not optimal, thus the expected length of the $V I H C_{G}$ code is greater. Based on this fact, and using the same reasoning as in [34, Theorem 5.8.1] the proof of the theorem is immediate.

\subsection{Compression algorithm based on VIHC}

This section presents the compression algorithm which employs the VIHC scheme for compressing the test set. In this work, both the compression of the difference vector sequence $\left(T_{d i f f}\right)$ and the compression of the initial test sequence $\left(T_{D}\right)$ is taken into account. The initial test set $\left(T_{D}\right)$ is partially specified and the test vectors can be reordered. This is because automated test pattern generator (ATPG) tools specify only a small number of bits in every test vector, thus allowing for greater flexibility during mapping. In addition, since full scan circuits are targeted, reordering of test vectors is also allowed. The mapping and reordering occurs in a pre-processing step, which prepares the test set for compression by mapping the "don't cares" to '0's or '1's and reordering the test set such that longer runs of '0's are generated. This is later on exploited by coding schemes based on runs of '0's leading to increased compression ratio. The compression algorithm has three main procedures: $i$ ) prepare initial test set, ii) compute Huffman code and iii) generate decoder information. The first two procedures are used for compression only, while the last one determines the decoding architecture described in Section 4. The compression algorithm is illustrated in Algorithm 1. The inputs to the algorithm are the initial test set $\left(T_{D}\right)$ and the type of performed compression (compression_type), which can be either diff, if the reordered difference vector sequence is compressed, or var, if the reordered initial test set is compressed. It is important to note that the MinTest dynamic compacted test sets [39] used in this paper have on average $27 \%$ specified bits, with up to $100 \%$ specified bits per test vector. 


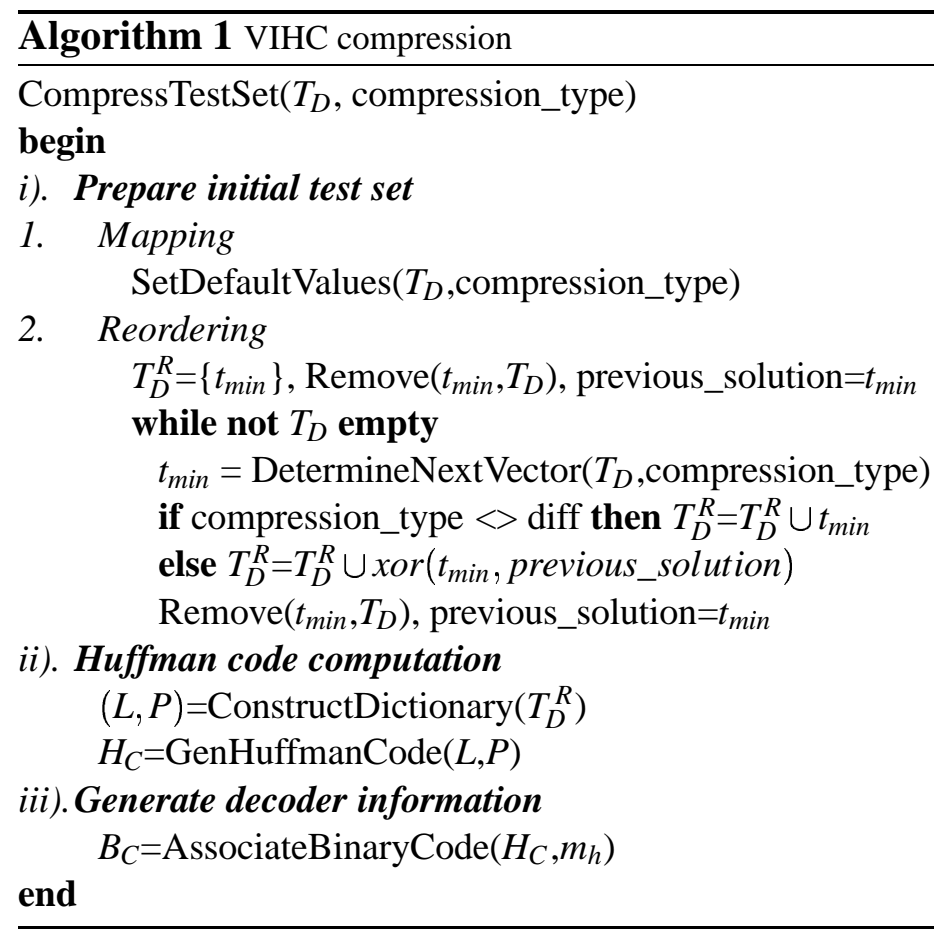

i) Prepare initial test set: As illustrated in the previous section the VIHC method uses runs of '0's as input patterns. The length of these patterns can be increased using this pre-processing procedure. The procedure consists of two steps. In the first step, for the compression of $T_{\text {diff }}$ the "don't cares" are mapped to the value of the previous vector on the same position; for the compression of $T_{D}$, the “don't cares" are mapped to '0's (SetDefaultValues in Algorithm 1). In the second step the test set is reordered and denoted by $T_{D}^{R}$ in the algorithm. The reordering process is illustrated in the algorithm in step 2. Starting with the test vector which has the minimum number of '1's, the next test vector $t_{\min }$ is determined (procedure DetermineNextVector in the algorithm) such that the following conditions are met. For $T_{d i f f}$, the next test vector is selected such that the number of '1's in the difference between the test vector and the previous_solution is minimum, and the length of the minimum run of '0's in the difference is maximum (i.e., if there are more test vectors which lead to the same number of '1's in the difference with previous_solution, then the one which has the minimum run of '0's of maximum length is selected). For $T_{D}$, the next test vector is selected such that the length of the minimum run of '0's is maximum. Further on, if the compression_type is diff, the difference between $t_{\min }$ and the previous solution (previous_solution) is added to the reordered test set $\left(T_{D}^{R}\right)$, otherwise, the selected test vector $\left(t_{\text {min }}\right)$ is added to $T_{D}^{R}$. The reordering algorithm has a complexity of $O\left(\left|T_{D}\right|^{2}\right)$, where $\left|T_{D}\right|$ represents 


\begin{tabular}{|c|c|c|}
\hline$m_{h}$ & $H_{C}$ & $B_{C}$ \\
\hline \hline 4 & 000 & $(001,0)$ \\
\cline { 2 - 3 } & 001 & $(010,0)$ \\
\cline { 2 - 3 } & 010 & $(011,0)$ \\
\cline { 2 - 3 } & 011 & $(100,0)$ \\
\cline { 2 - 3 } & 1 & $(100,1)$ \\
\hline
\end{tabular}

Table 1. Core user/vendor information exchange

the number of test vectors in the test set. The mapping and reordering performed in this procedure will be exploited by the variable-length input patterns used for Huffman code computation in the following procedure.

ii) Huffman code computation: Based on the chosen group size $\left(m_{h}\right)$ the dictionary of variablelength input patterns $L$ and the number of occurrences $P$ are determined from the reordered test set (ConstructDictionary in Algorithm 1). This is a mandatory step, because, in contrast to Golomb which has the codewords precomputed for a given group size, VIHC determines the codewords based on the group size and the pattern distribution (i.e., the group size determines the pattern distribution and the pattern distribution determines the Huffman codes). Using $L$ and $P$ the Huffman codes $\left(H_{C}\right)$ are computed using a Huffman algorithm [34] (GenHuffmanCode in the algorithm). Having determined the Huffman codes, the last procedure generates the information required to construct the decoder.

iii) Generate decoder information: This procedure is performed by AssociateBinaryCode in Algorithm 1 . For each Huffman code $c_{i}$ a binary code $b_{i}$ is assigned. The binary code is composed from the length of the initial pattern on $\left\lceil\log _{2}\left(m_{h}+1\right)\right\rceil$ bits and a special bit which identifies the cases when the initial pattern is composed of ' 0 's only, or it is a run of ' 0 's ending in 1 . Thus, $b_{i}=\left(\left|L_{i}\right|, 0\right)$ for $i<m_{h}$, and $b_{m_{h}}=\left(\left|L_{m_{h}}\right|, 1\right)\left(\left|L_{i}\right|\right.$ denotes the length of pattern $L_{i}$, see Section 3.1). It should be noted that if the core provider supplies a test set compressed with the VIHC method, the group size, the Huffman codes and the corresponding binary codes are the only information required to generate the on-chip decoder. For the example in Figure 2, Table 1 illustrates the required information. The following section shows how the binary code is used by the proposed VIHC decoder. 


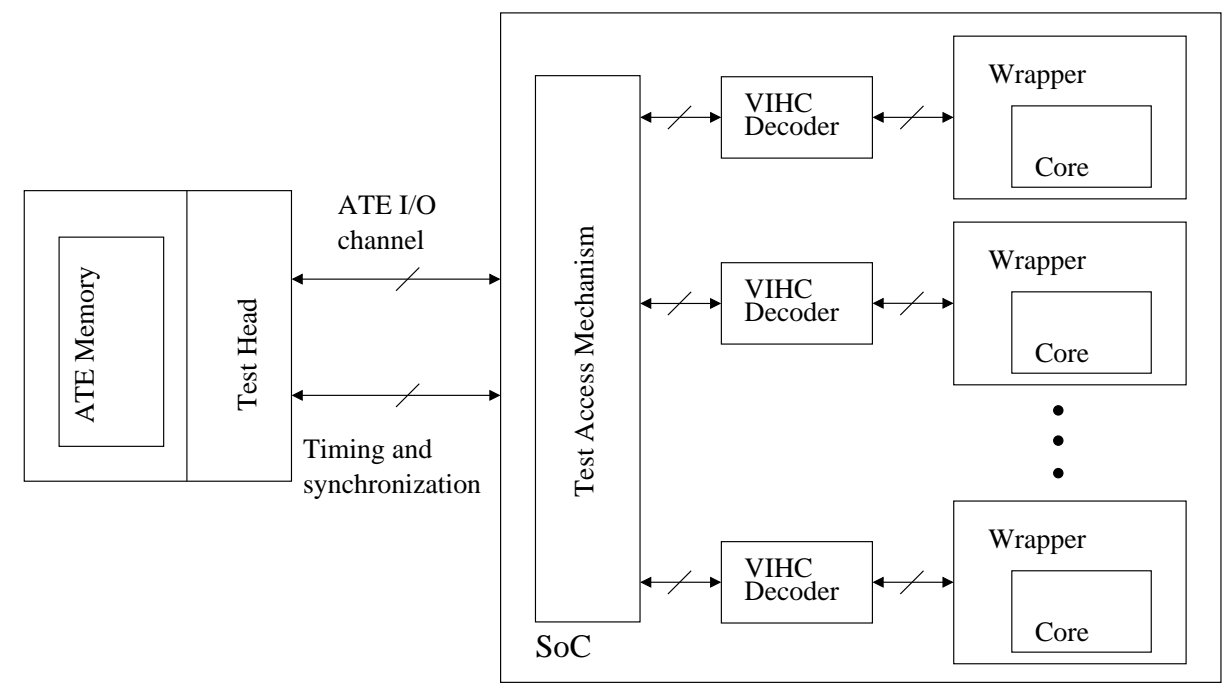

Figure 5. VIHC generic decompression architecture based on [2]

\section{Decompression}

This section introduces the new on-chip VIHC decoder (Section 4.1) for the VIHC scheme and provides the TAT analysis for the proposed decoder (Section 4.2). For the remainder of this paper a generic decompression architecture as shown in Figure 5 is assumed. The VIHC decoder, proposed in Section 4.1, uses a novel parallel approach in contrast to the previous Golomb [2] serial decoder. It should be noted that for the decompression of $T_{d i f f}$, a CSR architecture $[2,30]$ is used after the VIHC decoder in Figure 5. This work assumes that the ATE is capable of external clock synchronization [36].

\subsection{VIHC decoder}

A block diagram of the VIHC decoder is given in Figure 6. The decoder comprises a Huffman decoder [29] (Huff-decoder) and a Control and Generator Unit (CGU). The Huff-decoder is a finite state machine (FSM) which detects a Huffman code and outputs the corresponding binary code. The CGU is responsible for controlling the data transfer between the ATE and the Huff-decoder, generate the initial pattern and control the scan clock for the CUT. The data in line is the input from the ATE synchronous with the external clock (ATE clock). When the Huff-decoder detects a codeword, the code line is high and the binary code is output on the data lines. The special input to the CGU is used to differentiate between the two types of patterns, composed of '0's only $\left(L_{m_{h}}\right)$ and runs of '0's ending in $1\left(L_{0} \ldots L_{m_{h}-1}\right)$. After loading the code, the CGU generates the pattern (data out) and the internal scan clock for the 


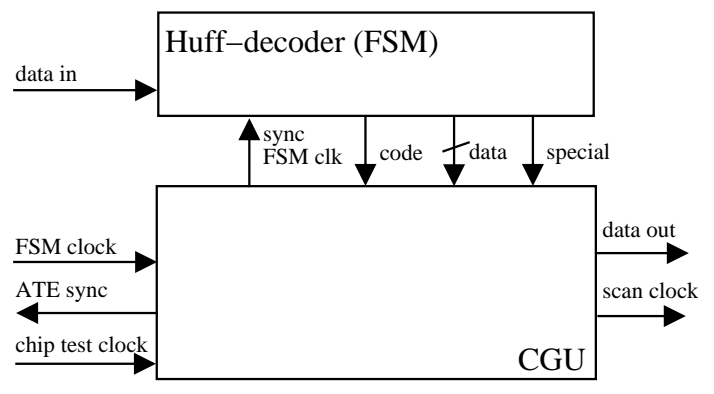

Figure 6. VIHC decoder

CUT. If the decoding unit generates a new code while the CGU is busy processing the current one, the ATE sync line is low notifying the ATE to stop sending data and the sync FSM clk is disabled forcing the Huff-decoder to maintain its current state. Dividing the VIHC decoder in Huff-decoder and CGU, allows the Huff-decoder to continue loading the next codeword while the CGU generates the current pattern. Thus, the Huff-decoder is interrupted only if necessary, which is in contrast to the Golomb [2] and the FDR [3] serial decoders. This leads to reduction in TAT when compared to the Golomb and the FDR methods, as it will be shown in Section 5. It should be noted that if the ATE responds to the stop signal with a given latency (i.e., it requires a number of clock cycles before the data stream is stopped or started), the device interface board between the ATE and the system will have to account for this latency using a first in first out (FIFO) - like structure. An additional advantage of the proposed on-chip parallel decoder is that having the two components working in two different clock domains (the Huff-decoder changes state with the ATE operating frequencies, and the CGU generates data at the on-chip test frequency), it will leverage the frequency ratio between the on-chip test frequency and the ATE operating frequency. This facilitates data delivery to the CUT at the on-chip test frequency using only one ATE channel, hence using temporal serialization (see Section 1.1) and therefore better resource usage.

The FSM for the Huffman decoder corresponding to the example from Figure 2 is illustrated in Figure 7. Starting from state $S_{1}$, depending on the value of data in the Huff-decoder changes its state. It is important to note the following:

- after the Huff-decoder detects a codeword it goes back to state $S 1$; for example, if the data in stream is 001 (first bit first) the Huff-decoder first changes its state from $S 1$ to $S 2$ then, from $S 2$ to $S 3$ after which back to $S 1$, and sets data and special to the corresponding binary code $((010,0)$ in this case), and the code line high;

- the number of ATE clock cycles needed to detect a code is equal to the length of the code; for 


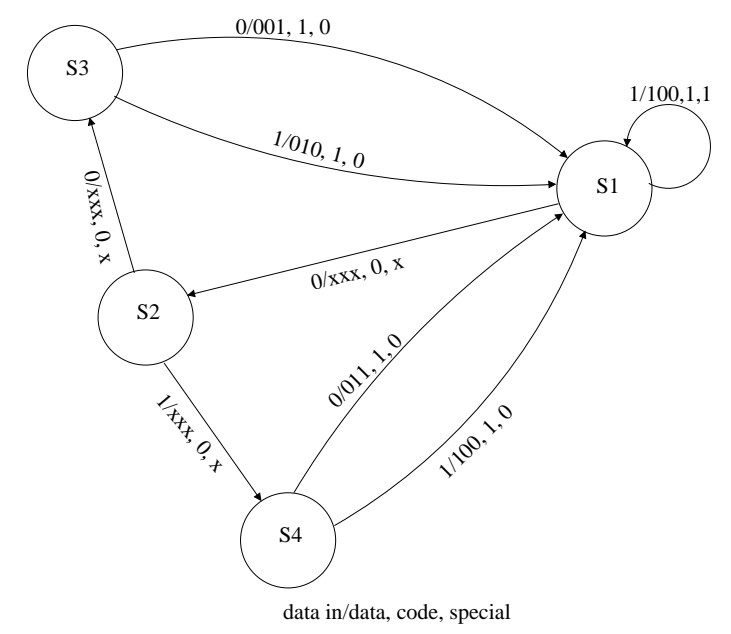

Figure 7. FSM for example in Figure 2

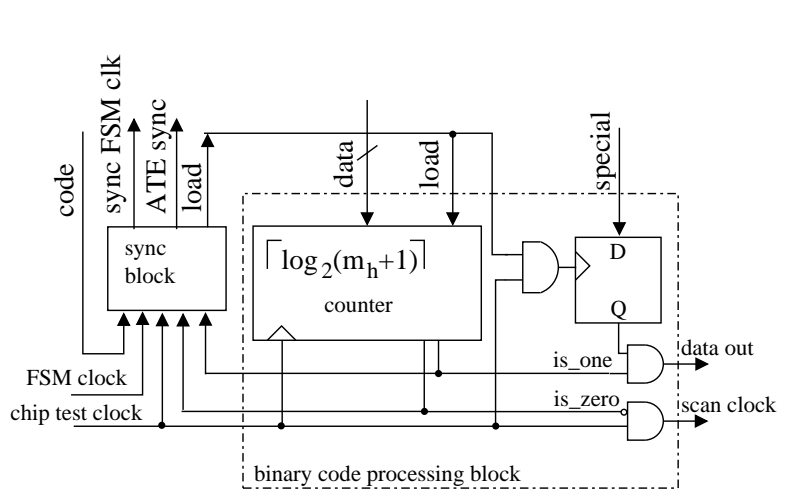

(a) $\mathrm{CGU}$

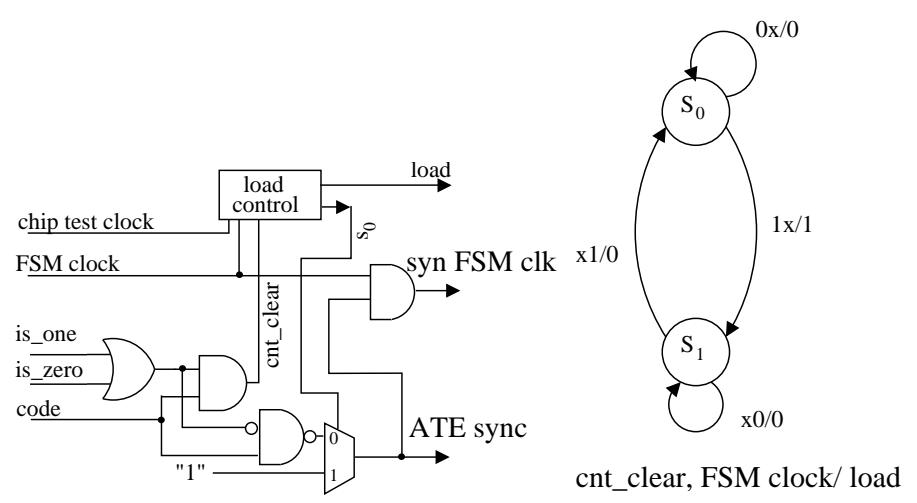

(b) Synchronization block (c) Load control FSM

Figure 8. CGU for VIHC decoder

example, if the data in stream is 001 (first bit first), the Huff-decoder needs three ATE clock cycles to identify the code;

- the Huff-decoder has a maximum of $m_{h}$ states for a group size of $m_{h}$; the number of states in a Huff-decoder is given by the number of leafs in the Huffman tree minus one; since there are a maximum of $m_{h}+1$ leafs for a group size of $m_{h}$, the number of states is $m_{h}$.

A high level view of the CGU is given in Figure 8(a). There are two main components of the CGU: the binary code processing block and the synchronization block. The binary code processing block comprises a counter which will hold the length of the initial pattern, and a flip flop which holds the special bit (see the binary code representation in Section 3.2). Being a parallel decoder, there are two 
issues to be considered. Firstly, the binary code processing block should be loaded only when a new code is identified; and secondly, the load of new data is done only when the pattern corresponding to the already loaded binary code was fully processed. These tasks are controlled by the synchronization block (sync) illustrated in Figure 8(b). As noted earlier, when the Huff-decoder identifies a code, it will output the associated binary code and set the code line high. When the code line is high, the sync block will determine the values of sync FSM clk, load and ATE sync. The key element in the synchronization block is the load control FSM, whose state diagram is detailed in Figure 8(c). The inputs into the load control FSM are the cnt_clear and the FSM clock. The cnt_clear will notify the FSM when the current code has been processed (i.e., the counter's value is either zero or one) and there is a new code available (i.e., the code line is high). The FSM clock is used to synchronize the load control FSM with the Huff-decoder FSM. When cnt_clear is 1 , the FSM will change to $S_{1}$ and set load to 1 . After one clock cycle, the FSM will set load to 0 . If FSM clock is 1 , the FSM will return to state $S_{0}$, otherwise it will remain in state $S_{1}$. This last condition was added since a new code can only occur after the FSM clock was 1 (this is how the Huff-decoder is controlled). When the FSM is in state $S_{0}$, the $S_{0}$ line in Figure $8(\mathrm{~b})$ together with the logic behind the multiplexer will ensure that the Huff-decoder continues the load of data from the ATE once the data has been loaded into the binary code processing block. Hence, stopping the stream of data from the ATE to the Huff-decoder only when necessary. The load control FSM can be implemented using one FF and additional logic. The FSM clock signal ensures that the FSM will reach a stable state after one internal clock cycle, therefore it has to be generated as an one internal clock cycle for each external clock cycle period. This can be easily achieved by using the same technique as in [36] for the serial scan enable signal.

When compared to the SC decoder [1], which has an internal buffer and a shift register of size $b$ (the group size), and a Huffman FSM of at least $b$ states, the proposed decoder has only a $\left\lceil\log _{2}\left(m_{h}+1\right)\right\rceil$ counter, two extra latches and a Huffman FSM of at most $m_{h}$ states. Thus, for the same group size $\left(m_{h}=b\right)$ significant reduction in area overhead is obtained, as it will be seen in Section 5. Similar to SC [1], the synchronization channel to the ATE can be eliminated if the VIHC codes are padded with dummy bits such that $w_{\min }$ increases. This however will affect the compression ratio. In addition, both, VIHC and SC, can eliminate the synchronization channel if the frequency ratio is greater or equal to their corresponding optimum frequency ratio. For VIHC this is illustrated in the following section. 


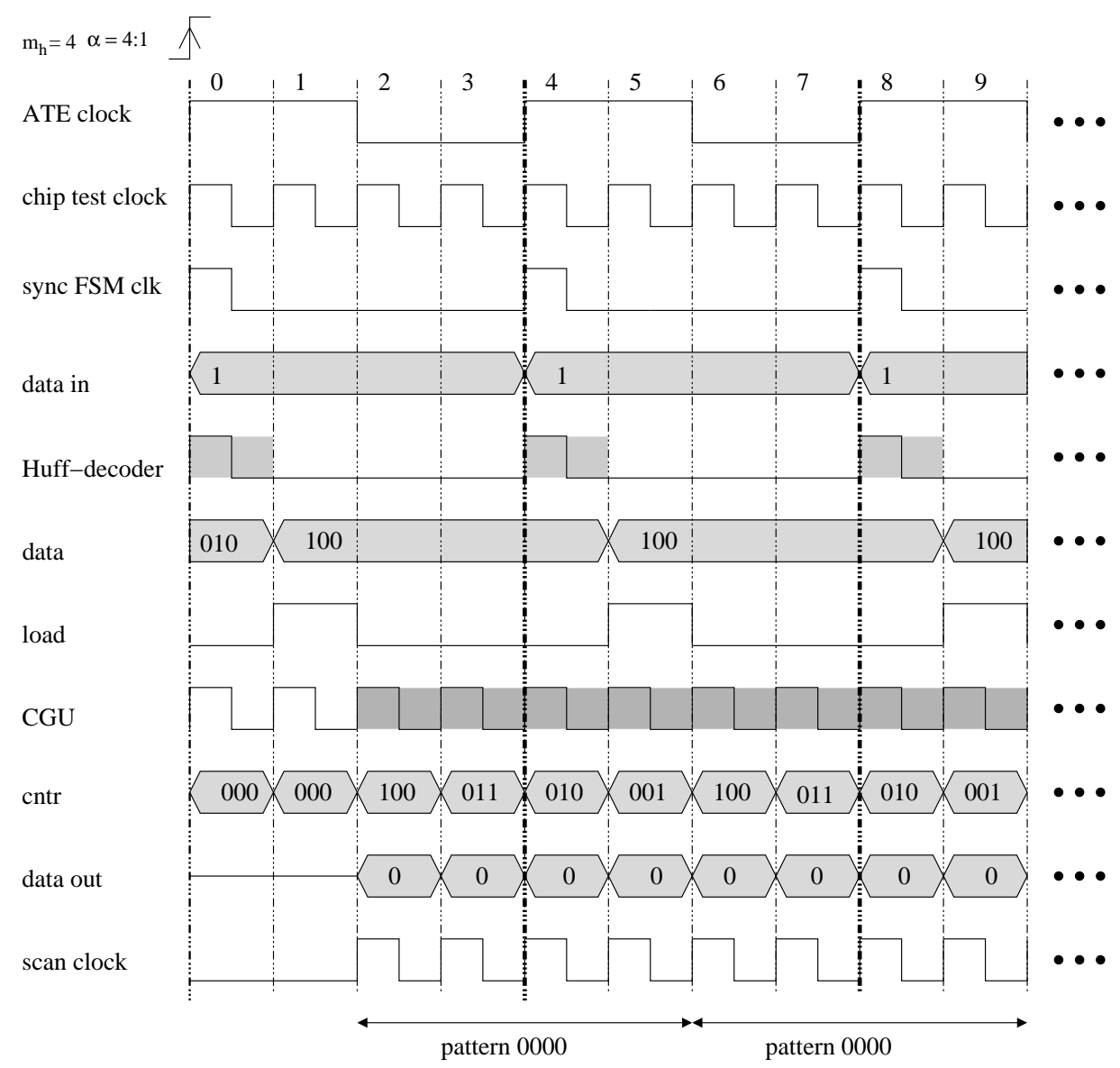

Figure 9. VIHC decoder timing diagram

\subsection{Test application time analysis}

Because the on-chip decoder has two components working in two different clock domains (i.e., the Huff-decoder is receiving data with ATE operating frequencies and the CGU generating data at the onchip test frequencies), the TAT is influenced by the frequency ratio between the two frequencies: the on-chip test frequencies and the ATE operating frequencies. To understand the effects of the frequency ratio on the TAT, this section provides a TAT analysis with respect to the frequency ratio. It is considered that the data is fed into the Huff-decoder FSM with the ATE operating frequency and that the FSM reaches a stable state after one on-chip test clock cycle. Analyzing the FSM for the Huffman decoder it can be observed that the number of ATE clocks needed to identify a codeword $c_{i}$ is equal to the size of the codeword $w_{i}$ (see Section 3.1). On the other hand, the number of internal clock cycles needed to generate a pattern is equal to the size of the pattern.

In order to illustrate the functionality of the CGU unit and to provide an example for the TAT analysis 
described next, Figure 9 shows the timing diagram for a frequency ratio of $\alpha=4$, considering $m_{h}=4$. The diagram corresponds to the generation process of the first two " 0000 " patterns in Figure 2 (see Example 1 in Section 3.1). This case was chosen to illustrate the parallelism of the proposed VIHC decoder and the overlap between generating the last bit of the pattern and loading the next binary code. The ATE clock, the chip test clock at a ratio of $4: 1$ with respect to the ATE clock, and the sync FSM clk required to drive the Huff-decoder are shown in the upper part of Figure 9. The data in row illustrates the data send from the ATE to the VIHC decoder. As detailed in the previous section, the Huff-decoder reaches a stable state after one internal clock cycle. Hence, the data signals which are loaded into the CGU unit are valid after one internal clock cycle. The time intervals in which the Huff-decoder identifies the last bit of a codeword and outputs the binary code (see Section 4.1) are highlighted in the Huff-decoder row with dashed areas. With the data signals valid, the CGU sets the load signal high and loads 100 into the counter (cntr row in Figure 9 clock cycle 2). For the next four chip test clock cycles, the CGU is busy generating pattern "0000", as illustrated in the figure with the dashed areas in row $C G U$. The cntr is decremented and the data-out outputs the patterns' bits. While cntr is not zero, the scan clk is generated. At clock cycle 4, the Huff-decoder will identify the next codeword. Again, the load signal is high and the data signals are loaded into the counter. It is important to note that this occurs simultaneously with the output of the last bit from the previous pattern. Hence, between two consecutive counter loads, for $\alpha=4$, there are effectively 4 clock cycles in which data can be generated.

Formally, if $\alpha=\frac{f_{\text {chip }}}{f_{\text {ate }}}$ is the ratio between the on-chip test frequency $\left(f_{\text {chip }}\right)$ and the ATE operating frequency $\left(f_{\text {ate }}\right)$, then after a Huffman code is identified, $\alpha-1$ internal clock cycles from the current ATE cycle can be used to generate a pattern. Thus, in order for the Huff-decoder to run without being stopped by the CGU, the CGU has to be able to generate the pattern $L_{i}$ in the number of internal clock cycles remained from the ATE clock in which it was started plus the number of internal clock cycles needed for the Huff-decoder to identify the next codeword. Or, $\left|L_{i}\right|<(\alpha-1)+1+\alpha *\left(w_{j}-1\right)$, where $w_{j}$ is the length of the next codeword in bits. With $\max \left(\left|L_{i}\right|\right)=m_{h}$ and $\min \left\{w_{i}\right\}=w_{\min }$, the frequency ratio to obtain the smallest TAT is given by $\alpha_{\text {max }}=\frac{f_{\text {chip }}}{f_{\text {ate }}}=\frac{m_{h}}{w_{\text {min }}}$ (optimum frequency ratio). The lowest TAT is given by $H\left(m_{h}\right)+\delta$, where $H\left(m_{h}\right)$ is the number of bits in the compressed test set in ATE clock cycles and $\delta$ is the number of extra ATE clocks needed to decode the last codeword. When the frequency ratio is greater than $\alpha_{\max }$, an increase in the compression ratio will lead to a reduction in test application 
time. Thus, an increase in frequency ratio leads to lower test application times when the compression ratio obtained by the proposed VIHC scheme is greater than the one obtained by SC [1], as it will be seen in Section 5.

To compute the TAT for the compressed test set for frequency ratios smaller than the optimum frequency ratio, an approximation function with respect to $\alpha=\frac{f_{\text {chip }}}{f_{\text {ate }}}$ is given next. The function to compute the TAT for the VIHC decoder in Section 4.1 is given by

$$
\tau(\alpha)=H\left(m_{h}\right)+\delta+\sum_{i=\left\lceil w_{\min } * \alpha\right\rceil}^{m_{h}} n_{i} *\left\lceil\frac{\left|L_{i}\right|-w_{\min } * \alpha}{\alpha}\right\rceil
$$

when each pattern is followed by the codeword with the lowest decoding time. In order to give an approximation to the above function the pattern distribution is analyzed. For example, for the full scan version of the s5378 ISCAS89 benchmark circuit, Figure 10 illustrates the pattern distribution for $m_{h}=$ 16 for the MinTest [39] test set. It can be observed that the patterns with length smaller than 4 and greater than $\left(m_{h}-1\right)$ are the most frequent. Therefore the function can be approximated with

$$
\tau(\alpha) \approx H\left(m_{h}\right)+\delta+n_{o} *\left\lceil\frac{m_{h}-w_{\min } * \alpha}{\alpha}\right\rceil
$$

where $n_{o}$ is the number of patterns with length $m_{h}$ (the patterns $L_{m_{h}-1}$ and $L_{m_{h}}$ ). It should be noted that $\delta$ will be ignored since $\delta \ll H\left(m_{h}\right)$. In the worst case scenario, for $n_{o}=\left[\frac{n}{m_{h}+1}\right]$ (each run of length $m_{h}$ is assumed to be followed by a run of length 1)

$$
\tau(\alpha) \approx H\left(m_{h}\right)+\frac{n}{m_{h}+1} * \frac{m_{h}-w_{\min } * \alpha}{\alpha}
$$

To show that formula (3) provides an approximation for the TAT of the decoder, in Figure 11 a comparison between the TAT obtained using the above formula and the TAT obtained by simulating the compressed test set for circuit s5378 using a group size of 16 is illustrated. The TAT reported in the figure is in ATE clock cycles. As it can be observed, the difference between the two curves is very small. It should also be noted that the TAT is minimum for a frequency ratio of 8 , since the minimum code length is $2\left(w_{\min }=2\right)$ in this case. It should be also noted that for frequency ratios greater than $\alpha_{\max }$ the TAT equals $\tau\left(\alpha_{\max }\right)$ and the synchronization channel which notifies the ATE to start/stop the data stream is no longer necessary. 


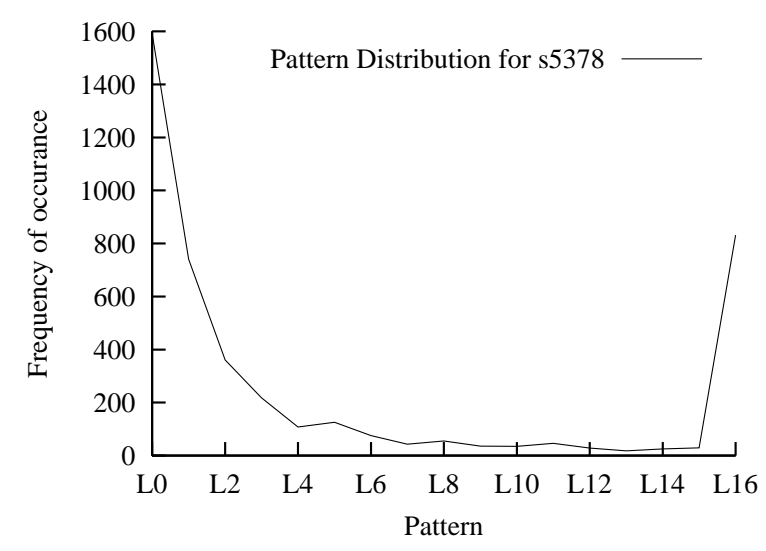

Figure 10. Pattern distribution for s5378 with $m_{h}=16$

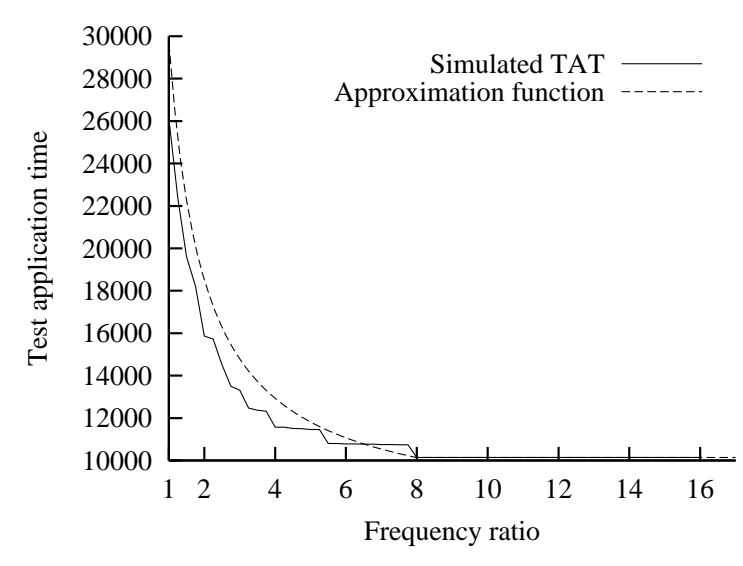

Figure 11. TAT for s5378 with $m_{h}=16$

\section{Experimental results}

To validate the efficiency of the proposed method, experiments were performed on the full-scan version of the largest ISCAS89 benchmark circuits [40]. The test sets used in these experiments were obtained using MinTest [39] dynamic compaction (also used in [2]). The experiments were performed on a Pentium III 500MHz workstation with 128 MB DRAM. SC [1], Golomb [2], FDR [3] and the proposed VIHC method were implemented in $\mathrm{C}++$. First, the proposed compression method is analyzed from the compression ratio's point of view. Secondly, an area overhead comparison between the four on-chip decoders (SC [1], Golomb [2], FDR [3] and the proposed VIHC method) is given. Finally, the TATs obtained by simulating the four methods' on-chip decoders are compared. The experiments were performed for both the difference vector sequence $\left(T_{\text {diff }}\right)$ and the initial test set $\left(T_{D}\right)$. Since SC [1] did not report compression ratios for the test sets used in this paper the results reported in this section were 
computed by implementing the SC method and applying it to the MinTest [39] test sets. It should be noted that SC was applied on the same test set as VIHC after the mapping and reordering algorithm proposed in this paper (see Section 3.2). In addition, we have considered the number of patterns used for selective coding as being given by the group size plus one, i.e., for a group size of $m_{h}=4,5$ patterns were considered. This is motivated by the pattern distribution obtained with the considered mapping and reordering algorithm which for $m_{h}=4$ usually leads to the patterns: "0000", "0001", "0010", "0100" and "1000", as being the most frequent.

Compression ratio Prior to providing a comparison with previous approaches, let's analyze the performances of the proposed method. The experiments performed using the $T_{\text {diff }}$ and $T_{D}$ test sets are summarized in Tables 2 and 3 respectively. The tables list, for each ISCAS89 benchmark circuit, the compression ratio obtained for different group sizes (columns 2 to 8), the maximum obtained compression (Max), the size of the maximum compressed test set $(H(M a x))$, the size of the initial test set in bits, and the size of the fully compacted MinTest [8] test set. As it can be observed in the tables, the maximum compression ratio is up to 86.83 for circuit s13207 in Table 2, and up to 83.51 for circuit s13207 in Table 3. Analyzing the compression ratios vs. group sizes in the tables, it can be easily observed that the compression ratio tends to increase with the increase in group size. This is illustrated in Figure 12 for circuit s5378. However, after a group size of 16, the increase in compression ratio up to the maximum compression ratio is less than $3 \%$. It is interesting to note that this is contrary to the Golomb [2] compression ratio vs. group size trend that tends to have a good compression ratio for one group size, after which by increasing the group size the compression ratio gets worse. This can be explained by the fact that the particular pattern distribution for which Golomb leads to an optimum code, and hence to good compression, is generally not respected (see Section 3.1).

In the following a comparison between the four compression methods (SC [1], Golomb [2], FDR [3] and the proposed VIHC method) is given. The comparison is summarized in Table 4. The table lists, the compression obtained with each method for the $T_{\text {diff }}$ and $T_{D}$ test sets. It can be easily observed that except for circuit s35932 in the case of $T_{D}$, the compression obtained by the proposed compression method leads to constant improvement in compression ratio. For example, for circuit s38417, in the case of $T_{d i f f}$, the proposed method obtains an increase in compression ratio of $11 \%, 22 \%$ and $13 \%$ over SC, Golomb [2] and FDR [3], respectively. Similarly, for circuit s38417 in the case of $T_{D}$, the increase in 


\begin{tabular}{|c|c|c|c|c|c|c|c|c|c|c|}
\hline \multirow[b]{3}{*}{ Circuit } & \multicolumn{7}{|c|}{ Compression ratio } & \multirow{3}{*}{$\begin{array}{l}\text { Size of } \\
H(\operatorname{Max})\end{array}$} & \multirow{3}{*}{$\begin{array}{c}\text { Size of } \\
T_{\text {diff }}\end{array}$} & \multirow{3}{*}{$\begin{array}{c}\text { Size of } \\
\text { MinTest } \\
{[8]}\end{array}$} \\
\hline & \multicolumn{6}{|c|}{ Group size } & \multirow[b]{2}{*}{ Max } & & & \\
\hline & 4 & 6 & 8 & 12 & 14 & 16 & & & & \\
\hline s5378 & 51.52 & 54.46 & 55.23 & 55.85 & 56.66 & 57.33 & 60.73 & 9328 & 23754 & 20758 \\
\hline s9234 & 1.84 & 58.12 & 58.75 & 58.45 & 58.76 & 59.02 & 60. & 153 & 39273 & 25935 \\
\hline s13207 & 69.02 & 75.90 & 79.07 & 82.03 & 82.69 & 83.21 & $\overline{86.83}$ & 21758 & 165200 & 163100 \\
\hline s15850 & 60.69 & 65.67 & 67.48 & 68.65 & 68.86 & 68.99 & 72.34 & 21291 & 76986 & 57434 \\
\hline $\mathrm{s} 35932$ & 40.35 & 49.92 & 56.97 & 61.08 & 62.54 & 66.47 & 71.91 & 7924 & 28208 & 19393 \\
\hline s38417 & 54.51 & 58.57 & 59.96 & 60.86 & 61.22 & 61.98 & 66.38 & 55387 & 164736 & 113152 \\
\hline s38584 & 56.97 & 61.21 & 62.50 & 63.01 & 63.00 & 62.97 & 66.29 & $\overline{67114}$ & 199104 & 104111 \\
\hline
\end{tabular}

Table 2. VIHC for $T_{\text {diff }}$

\begin{tabular}{|c|c|c|c|c|c|c|c|c|c|c|}
\hline \multirow[b]{3}{*}{ Circuit } & \multicolumn{7}{|c|}{ Compression ratio } & \multirow{3}{*}{$\begin{array}{c}\text { Size of } \\
H(\text { Max })\end{array}$} & \multirow{3}{*}{$\begin{array}{c}\text { Size of } \\
T_{D}\end{array}$} & \multirow{3}{*}{$\begin{array}{c}\text { Size of } \\
\text { MinTest } \\
{[8]}\end{array}$} \\
\hline & \multicolumn{6}{|c|}{ Group size } & \multirow[b]{2}{*}{ Max } & & & \\
\hline & 4 & 6 & 8 & 12 & 14 & 16 & & & & \\
\hline $\begin{array}{l}\mathrm{s} 5378 \\
\end{array}$ & 41.13 & 42.15 & 42.85 & 44.85 & 45.73 & 46.94 & 51.78 & 11453 & 23754 & 20758 \\
\hline s9234 & 45.27 & 46.14 & 45.27 & 46.14 & 46.02 & 46.14 & 47.25 & 20716 & 39273 & 25935 \\
\hline s13207 & 67.60 & 74.12 & 76.92 & 79.49 & 80.03 & 80.36 & 83.51 & 27248 & 165200 & 163100 \\
\hline s15850 & 58.01 & 62.43 & 63.73 & 64.42 & 64.28 & 64.06 & 67.94 & 24683 & 76986 & 57434 \\
\hline s35932 & 29.76 & 38.73 & 44.24 & 47.07 & 47.89 & 51.84 & 56.08 & 12390 & 28208 & 19393 \\
\hline s38417 & 37.19 & 40.45 & 43.78 & 46.97 & 47.34 & 47.79 & 53.36 & 76832 & 164736 & 113152 \\
\hline s38584 & 54.43 & 57.89 & 58.81 & 58.90 & 59.17 & 59.62 & 62.28 & 75096 & 199104 & 104111 \\
\hline
\end{tabular}

Table 3. VIHC for $T_{D}$

compression ratio is $8 \%, 15 \%$ and $10 \%$ over SC, Golomb [2] and FDR [3], respectively. It should be noted that in the case of circuit s35932, for the $T_{D}$ test set, the SC [1] obtains better compression because of the regular nature of the test set, however, this is an isolated case, as it can be seen in the table. To illustrate the improvement in compression ratio when using the proposed reordering algorithm over the one proposed in [2], the table also illustrates the compression ratios obtained when applying Golomb and FDR on the test sets mapped and reordered with the algorithm given in Section 3.2. These results are given in the table under the $T_{D}^{R}$ heading for Golomb and FDR in the case of $T_{\text {diff }}$ and $T_{D}$ respectively. As it can be seen, in the case of $T_{\text {diff }}$ the increase in compression ratio when compared to the results reported in [2] and [3] for the two methods is considerable. For example, for circuit s35932, 23.28\% and $39.59 \%$ improvement in compression ratio is obtained. In the case of $T_{D}$, the increase in compression ratio is small or negligible. This is because, on average, the distribution of the runs of '0's in the case of $T_{D}$ is dependent mainly on the mapping, while the reordering has smaller influence. In order to provide a fair comparison, we will use the results obtained using the proposed mapping and reordering algorithm 


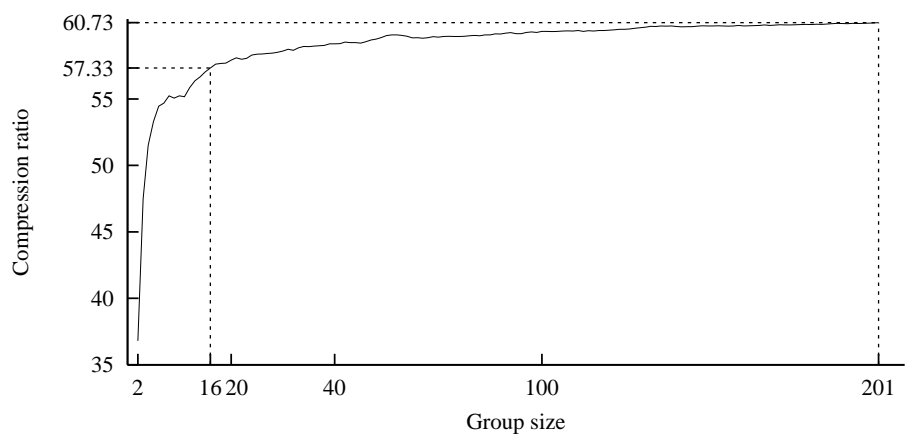

Figure 12. Compression ratio vs. group size for s5378 with $T_{\text {diff }}$

\begin{tabular}{|c|c|c|c|c|c|c|c|c|c|c|c|c|}
\hline \multirow[b]{3}{*}{ Circuit } & \multicolumn{6}{|c|}{$\overline{T_{\text {diff }}}$} & \multicolumn{6}{|c|}{10} \\
\hline & \multirow{2}{*}{$\begin{array}{l}\mathrm{SC} \\
{[1]}\end{array}$} & \multicolumn{2}{|c|}{ Golomb } & \multicolumn{2}{|c|}{ FDR } & \multirow[b]{2}{*}{ VIHC } & \multirow{2}{*}{$\begin{array}{l}\mathrm{SC} \\
{[1]}\end{array}$} & \multicolumn{2}{|c|}{ Golomb } & \multicolumn{2}{|c|}{ FDR } & \multirow[b]{2}{*}{ VIHC } \\
\hline & & [2] & $T_{D}^{R}$ & [3] & $T_{D}^{R}$ & & & [3] & $T_{D}^{R}$ & [3] & $T_{D}^{R}$ & \\
\hline s5378 & 2.33 & 40.70 & 51.00 & 8.19 & 9.00 & 60.73 & 43.64 & $\overline{37.11}$ & $\overline{37.1}$ & 48.02 & 8.03 & 1.78 \\
\hline s9234 & 2.63 & 43.34 & 58.08 & 44.88 & 58.85 & 60.96 & 40.04 & 45.25 & 45.27 & 43.59 & 43.53 & 47.25 \\
\hline s13207 & 7.73 & 74.78 & 82.34 & 78.67 & 85.50 & 86.83 & 74.43 & 79.74 & 79.75 & 81.30 & 81.30 & 83.51 \\
\hline s15850 & 3.49 & 47.11 & 66.58 & 52.87 & 71.02 & 72.34 & 58.84 & 62.82 & 62.83 & 66.22 & 66.23 & 67.94 \\
\hline $\mathrm{s} 35932$ & 5.72 & N/A & 23.28 & 10.19 & 49.78 & 71.91 & 64.64 & N/A & N/A & 19.37 & 19.36 & 56.08 \\
\hline s38417 & 57.26 & 44.12 & 56.72 & 54.53 & 64.32 & 66.38 & 45.15 & 28.37 & 28.38 & 43.26 & 43.26 & 53.36 \\
\hline s38584 & 58.74 & 47.71 & 61.20 & 52.85 & 65.27 & 66.29 & 55.24 & 57.17 & 57.17 & 60.91 & 60.92 & 62.28 \\
\hline
\end{tabular}

Table 4. Best compression comparison

for the remainder of this paper. Since, as illustrated in Figure 12, the increase in compression ratio for group sizes larger than 16 is small (less than $3 \%$ on average), further on the maximum group size for all the comparisons is considered 16.

To show that the proposed method exploits better the test set for a given group size, in the following a comparison between the SC, Golomb and VIHC is given, for the group sizes for which SC and Golomb obtain the best compression ratio for a group size of maximum 16. The comparison between SC and VIHC is given in Table 5 and the comparison between Golomb and VIHC is given in Table 6. Both tables list the group size and the compression ratio for which the corresponding method obtained best compression for the $T_{\text {diff }}$ and $T_{D}$ test sets. Analyzing Table 5, it can be easily found that, for the $T_{\text {diff }}$ test set, the proposed compression method (VIHC) obtains always better compression ratios than SC. For the $T_{D}$ test set, with the exception of the s35932 circuit, the VIHC compression method always obtains better compression ratios. When compared to Golomb [2] (see Table 6), the proposed VIHC compression method obtains always better compression ratios. The improvements are up to $20 \%$ for $T_{D}$ in the case of s35932, and up to $29 \%$ for $T_{\text {diff }}$ for the same circuit. These comparisons have illustrated 


\begin{tabular}{|c||c||c|c||c||c|c|}
\hline \multicolumn{1}{|c||}{} & \multicolumn{3}{c||}{$T_{\text {diff }}$} & \multicolumn{3}{c|}{$T_{D}$} \\
\cline { 2 - 7 } Circuit & Group & SC & & Group & SC & \\
size & {$[1]$} & VIHC & size & {$[1]$} & VIHC \\
\hline \hline s5378 & 12 & 52.33 & $\mathbf{5 5 . 8 5}$ & 12 & 43.64 & $\mathbf{4 4 . 8 5}$ \\
\hline s9234 & 10 & 52.63 & $\mathbf{5 8 . 7 9}$ & 8 & 40.04 & $\mathbf{4 5 . 2 7}$ \\
\hline s13207 & 16 & 77.73 & $\mathbf{8 3 . 2 1}$ & 16 & 74.43 & $\mathbf{8 0 . 3 6}$ \\
\hline s15850 & 16 & 63.49 & $\mathbf{6 8 . 9 9}$ & 16 & 58.84 & $\mathbf{6 4 . 0 4}$ \\
\hline s35932 & 16 & 65.72 & $\mathbf{6 6 . 4 7}$ & 16 & $\mathbf{6 4 . 6 4}$ & 51.84 \\
\hline s38417 & 12 & 57.26 & $\mathbf{6 0 . 8 6}$ & 10 & 45.15 & $\mathbf{4 5 . 2 5}$ \\
\hline s38584 & 14 & 58.74 & $\mathbf{6 3 . 0 0}$ & 12 & 55.24 & $\mathbf{5 8 . 9 0}$ \\
\hline
\end{tabular}

Table 5. Comparison between SC and VIHC

\begin{tabular}{|c|c||c|c||c||c|c|}
\hline & \multicolumn{3}{|c||}{$T_{\text {diff }}$} & \multicolumn{3}{c|}{$T_{D}$} \\
\cline { 2 - 7 } Circuit & $\begin{array}{c}\text { Group } \\
\text { size }\end{array}$ & $\begin{array}{c}\text { Golomb } \\
T_{D}^{R}\end{array}$ & VIHC & $\begin{array}{c}\text { Group } \\
\text { size }\end{array}$ & $\begin{array}{c}\text { Golomb } \\
T_{D}^{R}\end{array}$ & VIHC \\
\hline \hline s5378 & 8 & 51.00 & $\mathbf{5 5 . 2 3}$ & 4 & 37.13 & $\mathbf{4 1 . 1 3}$ \\
\hline s9234 & 8 & 58.08 & $\mathbf{5 8 . 7 5}$ & 4 & 45.27 & $\mathbf{4 5 . 2 7}$ \\
\hline s13207 & 16 & 82.34 & $\mathbf{8 3 . 2 1}$ & 16 & 79.75 & $\mathbf{8 0 . 3 6}$ \\
\hline s15850 & 8 & 66.58 & $\mathbf{6 7 . 4 8}$ & 8 & 62.83 & $\mathbf{6 3 . 7 3}$ \\
\hline s35932 & 4 & 23.28 & $\mathbf{4 0 . 3 5}$ & 4 & N/A & $\mathbf{2 9 . 7 6}$ \\
\hline s38417 & 8 & 56.72 & $\mathbf{5 9 . 9 6}$ & 4 & 28.38 & $\mathbf{3 7 . 1 9}$ \\
\hline s38584 & 8 & 61.20 & $\mathbf{6 2 . 5 0}$ & 8 & 57.17 & $\mathbf{5 8 . 8 1}$ \\
\hline
\end{tabular}

Table 6. Comparison between Golomb and VIHC

that for a given group size the proposed compression method yields better compression ratios, and hence it exploits better the test set than the previous approaches. It should be noted that in the case of SC, better results might be obtained at the cost of extra computational complexity if other mapping algorithms are used. In the following an area overhead comparison between the proposed on-chip decoder and the three previously proposed on-chip decoders (SC [1], Golomb [2], FDR [3]) is given.

Area overhead The four on-chip decoders were synthesized using the Synopsys Design Compiler [41]. The results are summarized in Table 7. For all methods, the entire decoder including buffers, shift registers and counters was synthesized. For SC, VIHC and Golomb, the area overhead was computed for a group size of 4,8 and 16. Without loss of generality the decoders for s5378 were synthesized. As shown in Table 7, the area overhead for SC is up to 3 times greater than the area overhead for VIHC. The area overhead for Golomb is almost equal to the one of VIHC, and the area overhead for FDR is constantly greater than the area overhead for VIHC. It is important to note that even though the Golomb 


\begin{tabular}{|c|c|c|c|}
\hline \multirow{3}{*}{$\begin{array}{c}\text { Compression } \\
\text { Method }\end{array}$} & \multirow{2}{*}{\multicolumn{3}{|c|}{$\frac{\text { Area overhead in } \mathrm{tu}^{*}}{\text { Group size }}$}} \\
\hline & & & \\
\hline & 4 & 8 & 16 \\
\hline 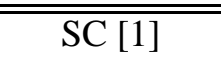 & 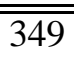 & 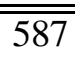 & 900 \\
\hline Golomb [2] & 125 & 227 & 307 \\
\hline FDR [3] & \multicolumn{3}{|c|}{320} \\
\hline VIHC & 136 & 201 & 296 \\
\hline
\end{tabular}

Table 7. Area overhead comparison for s5378

decoder [2] has lower area overhead for a group size of 4, when compared to the proposed decoder, by increasing the group size the area overhead of the Golomb's decoder is greater than the proposed one's. This is because, in order to decode the tail part of the code, the Golomb decoder basically implements the behavior of a counter within the Golomb FSM. It should be noted that for the case when the $T_{\text {diff }}$ test set is used, a CSR architecture is required. Since the CSR has to be of the length of the scan chain fed by the decoder, the CSR overhead is dominated by the length of the internal scan chain of the targeted core (e.g., for core s5378 the CSR comprises 179 scan cells), being independent of the chosen compression method. Hence, a CSR is needed by all four approaches and therefore not considered in the area overhead comparison given in Table 7. In addition, it has been shown in [30] how the internal scan chains of neighbouring cores in a SOC can be used to eliminate the need for the CSR. The comparisons provided until now show that the proposed method improves on the first two TDCE parameters: compression ratio and area overhead. In the following, the last parameter, the TAT, is compared with the previous work.

Test application time To provide an accurate TAT comparison between the four methods, let's consider the following experimental setup: the maximum frequency ratio is $\alpha=8$ and the maximum acceptable group size is 16 . To compute the TAT, a simulator was implemented based on the TAT analysis for SC [1], Golomb [2], FDR [3] and the VIHC decoder (Section 4.1). For all decoders it was assumed that the data is fed into the decoder at ATE operating frequency and the internal FSM reaches a stable state after one internal clock cycle. In order to provide a fair comparison, we first mapped and reordered the test sets, applied the compression methods and on the compressed test sets we performed the simulations for each method. The TATs resulted after the simulation are reported in Table 8. In addition the table also illustrates the TAT obtained for the fully compacted MinTest test set [8]. It should be noted that the TAT for the MinTest test set was considered to be the time needed to transfer the data to 


\begin{tabular}{|c|c|c|c|c|c|c|c|c|c|}
\hline \multirow[t]{2}{*}{ Circuit } & \multirow{2}{*}{$\begin{array}{l}\text { Comp. } \\
\text { Method }\end{array}$} & \multicolumn{4}{|c|}{ TAT (ATE clock cycles) for $T_{d i f f}$} & \multicolumn{4}{|c|}{ TAT (ATE clock cycles) for $T_{D}$} \\
\hline & & $\alpha=2$ & $\alpha=4$ & $\alpha=6$ & $\alpha=8$ & $\alpha=2$ & $\alpha=4$ & $\alpha=6$ & $\alpha=8$ \\
\hline \multirow[t]{5}{*}{ s5378 } & " MinTest [8] & \multicolumn{4}{|c|}{20758} & \multicolumn{4}{|c|}{20758} \\
\hline & SC [1] & 15835 & 12412 & 11323 & 11323 & 17259 & 14323 & 13387 & 13387 \\
\hline & Golomb [2] & 20649 & 16020 & 13782 & 13782 & 23529 & 19232 & 14935 & 14935 \\
\hline & $\begin{array}{l}\text { FDR [3] } \\
\end{array}$ & 22263 & 14678 & 12968 & 11679 & 24933 & 16803 & 15259 & 14039 \\
\hline & VIHC & 15868 & 11569 & 10777 & 10137 & 17668 & 13740 & $\overline{12914}$ & 12782 \\
\hline \multirow[t]{5}{*}{ s9234 } & MinTest [8] & \multicolumn{4}{|c|}{25935} & \multicolumn{4}{|c|}{25935} \\
\hline & SC [1] & 24815 & 20675 & 18605 & 18605 & 27459 & 23547 & 23547 & 23547 \\
\hline & Golomb [2] & 31186 & 23555 & 19894 & 19894 & 35580 & 28537 & 21494 & 21494 \\
\hline & $\begin{array}{l}\text { FDR [3] } \\
\end{array}$ & 36135 & 24128 & 21381 & 19001 & 42066 & 29206 & 26675 & 24086 \\
\hline & VIHC & 24895 & 17994 & 16905 & 16095 & 27235 & 23860 & 21154 & 21154 \\
\hline \multirow[t]{5}{*}{ s13207 } & MinTest [8] & \multicolumn{4}{|c|}{$\overline{163100}$} & \multicolumn{4}{|c|}{163100} \\
\hline & SC [1] & 89368 & 53490 & 45137 & 36784 & 91369 & 57455 & 49847 & $\overline{42239}$ \\
\hline & Golomb [2] & 104440 & 66381 & 47783 & 47481 & 106169 & 69190 & 51272 & 50847 \\
\hline & FDR [3] & 107059 & 63011 & 49858 & 41989 & 116101 & 70361 & 57089 & 48538 \\
\hline & VIHC & 89865 & 52769 & 44180 & 36065 & 90920 & 55229 & 47319 & 40048 \\
\hline \multirow[t]{5}{*}{ s15850 } & "MinTest [8] & \multicolumn{4}{|c|}{ ב57434 } & \multicolumn{4}{|c|}{$\overline{57434}$} \\
\hline & SC [1] & 47256 & 33844 & 30975 & 28106 & 47186 & 35432 & 31687 & 31687 \\
\hline & Golomb [2] & 57860 & 41452 & 33442 & 33442 & 64730 & 48528 & 32326 & 32326 \\
\hline & FDR [3] & 62419 & 39628 & 33767 & 29488 & 65020 & 42270 & 36732 & 32362 \\
\hline & VIHC & 47366 & 32513 & 29437 & 26692 & 48169 & 34735 & 31055 & 30871 \\
\hline \multirow[t]{5}{*}{ s35932 } & "MinTest [8] & \multicolumn{4}{|c|}{19393} & \multicolumn{4}{|c|}{19393} \\
\hline & SC [1] & 16870 & 11749 & 10710 & 9671 & 16879 & 11932 & $\mathbf{1 0 7 8 0}$ & 9974 \\
\hline & Golomb [2] & 31758 & 26699 & 21640 & 21640 & 42267 & 38341 & 34415 & 34415 \\
\hline & FDR [3] & 32509 & 20605 & 18438 & 17045 & 42159 & 27468 & 25893 & 24821 \\
\hline & VIHC & 17584 & 12076 & 10857 & 9645 & 19908 & 15641 & 14670 & 13736 \\
\hline \multirow[t]{5}{*}{ s38417 } & "MinTest [8] & \multicolumn{4}{|c|}{113152} & \multicolumn{4}{|c|}{113152} \\
\hline & $\mathrm{SC}[1]$ & 105076 & 78851 & 70403 & 70403 & 116282 & 98849 & 90354 & 90354 \\
\hline & Golomb [2] & 136554 & 103140 & 86974 & 86974 & 177074 & 147530 & 117986 & 11798 \\
\hline & FDR [3] & 144811 & 93450 & 81578 & 73182 & 186261 & 123700 & 113451 & 10521 \\
\hline & VIHC & 104642 & 74293 & 67940 & 62625 & 118989 & 92777 & 87914 & 87002 \\
\hline \multirow[t]{5}{*}{ "s38584 } & "MinTest [8] & \multicolumn{4}{|c|}{104111} & \multicolumn{4}{|c|}{104111} \\
\hline & SC [1] & 125141 & 97892 & 90020 & 82148 & 126309 & 98019 & 89124 & 89124 \\
\hline & Golomb [2] & 156238 & 115731 & 96073 & 96073 & 161236 & 122107 & 103200 & 10320 \\
\hline & FDR [3] & 170143 & 110982 & 96677 & 85687 & 179530 & 118628 & 104630 & 93260 \\
\hline & VIHC & 126969 & 92632 & 83130 & 82582 & 127849 & 92377 & 85783 & 80392 \\
\hline
\end{tabular}

Table 8. TAT comparison 
the core under test, hence the size of the test set in bits. Analyzing columns 3 to 6 and 7 to 10 , it can be observed that in general for small frequency ratios the SC has slightly better TAT than the proposed method (e.g., s5378, s15850 and s38584 with $\alpha=2$ for both $T_{d i f f}$ and $T_{D}$ ). However, generally the TATs of the proposed method are better than the ones of the previous methods (SC [1], Golomb [2], FDR [3]). Exception makes the circuit s35932 in the case of SC. This is because the minimum codeword $\left(w_{\min }\right)$ (see Section 4.2) for SC is 2 and $w_{\min }$ for VIHC is 1, and the compression ratio obtained by SC is almost equal to VIHC's in the case of $T_{d i f f}$, and greater than VIHC's in the case of $T_{D}$. These two combined lead to smaller TAT in this case. The reduction in TAT when compared to previous methods is detailed next. It can be observed that overall, in comparison to SC, TAT reduction of up to $12 \%$ is obtained for $T_{\text {diff }}$ (e.g., in the case of circuit s9234 for $\alpha=4$ ). Similarly for $T_{D}$, the TAT is reduced by up to $10 \%$ when compared to SC (e.g., in the case of circuit s9234 for $\alpha=6$ ). When compared to Golomb and FDR, for the $T_{\text {diff }}$ test set, TAT reductions up to $54 \%$ and $45 \%$ are obtained in the case of circuit s35932. For $T_{D}$, the TAT reduction is as high as $59 \%$ and $43 \%$ for Golomb and FDR respectively. For the rest of the circuits, in the case of $T_{d i f f}$, the TAT ranges from similar values (when frequency ratio increases) to reduction of up to $27 \%$ when compared to Golomb (s38417), and reduction of up to $31 \%$ when compared to FDR (s9234). The same applies for $T_{D}$, where the TAT reduction is up to $26 \%$ (s38417) when compared to Golomb, and up to $36 \%$ (s38417) when compared to FDR. Comparing the TAT with the one obtained by MinTest [8] gives an idea on how the TAT behaves for different frequency ratios when compared to fully compacted test sets. As it can be easily seen in the table, when compared to MinTest, the TAT is reduced as much as $77 \%$ for the circuit s13207 in the case of $T_{\text {diff }}$ for a frequency ratio of $\alpha=8$. Similarly for $T_{D}$ in the case of circuit s13207, TAT reduction up to $77 \%$ is obtained for $\alpha=8$. It should be noted that MinTest should improve its TAT if serialization buffers are introduced between the ATE and the SOC. However this implies the use of multiple ATE channels for one scan channel, which is avoided by the proposed approach.

Finally the comparisons conducted in this section between the previous compression methods [1-3] and the proposed VIHC are summarized in Table 9. While FDR [3] gives compression ratios comparable to VIHC, it leads to both higher TAT and greater area overhead. The main reason that TAT is a disadvantage for the FDR method is that, having no group size, the method requires a very high frequency ratio for which the minimum TAT is obtained (see Section 2). On the other hand, Golomb [2] has similar 


\begin{tabular}{c||c|c|c|c} 
& SC[1] & Golomb[2] & FDR[3] & VIHC \\
\hline \hline Compression & $X$ & $X$ & $\sqrt{ }$ & $\sqrt{ }$ \\
\hline Area overhead & $X$ & $\sqrt{ }$ & $X$ & $\sqrt{ }$ \\
\hline Test application time & $\sqrt{ }$ & $X$ & $X$ & $\sqrt{ }$ \\
\hline
\end{tabular}

Table 9. Previous approaches compared to VIHC

area overhead when compared to VIHC at the expense of lower compression ratio and higher TAT. SC [1], on the other hand, has overall comparable TAT when compared to VIHC. However, this is achieved at a very high penalty in area overhead which is the main shortcoming of the parallel decoder based on fixed-length Huffman coding.

\section{Concluding remarks}

This paper has presented a new compression method called Variable-length Input Huffman Coding (VIHC). This paper also provides a taxonomy on the factors which influence the three test data compression environment parameters: compression ratio, area overhead and test application time. Unlike previous approaches [1-3] which reduce some test parameters at the expense of the others, the proposed compression method is capable of minimizing all the three test data compression parameters simultaneously. This is achieved by accounting for multiple interrelated factors that influence the results, such as pre-processing the test set, the size and the type of the input patterns to the coding algorithm, and the type of the decoder. The results in Section 5 show that the proposed method obtains constantly better compression ratios than [1-3]. Furthermore, by exploiting the variable-length input approach, great savings in area overhead are achieved (up to threefold reduction when compared to the fixed-length approach [1]). Moreover, the parallel decoder leads to significant savings in TAT when compared to the serial decoders $[2,3]$. Thus, this paper has shown that the proposed method decreases the ATE memory and channel capacity requirements by obtaining good compression ratios, and reduces TAT through its parallel on-chip decoder with low area overhead.

Acknowledgments The authors wish to acknowledge Anshuman Chandra and Dr. Krishnendu Chakrabarty from Duke University for providing the test sets used in their papers. Thanks are also due to the anonymous reviewers for their constructive comments. 


\section{References}

[1] A. Jas, J. Ghosh-Dastidar, and N. A. Touba, "Scan Vector Compression/Decompression Using Statistical Coding," in Proceedings IEEE VLSI Test Symposium (VTS), pp. 114-121, IEEE Computer Society Press, Apr. 1999.

[2] A. Chandra and K. Chakrabarty, "System-on-a-Chip Test Data Compression and Decompression Architectures Based on Golomb Codes," IEEE Transactions on Computer-Aided Design, vol. 20, pp. 113-120, Mar. 2001.

[3] A. Chandra and K. Chakrabarty, "Frequency-Directed Run-Length (FDR) Codes with Application to System-on-a-Chip Test Data Compression," in Proceedings IEEE VLSI Test Symposium (VTS), pp. 114-121, IEEE Computer Society Press, Apr. 2001.

[4] E. J. Marinissen, Y. Zorian, R. Kapur, T. Taylor, and L. Whetsel, “Towards a Standard for Embedded Core Test: An Example," in Proceedings IEEE International Test Conference (ITC), (Atlantic City, NJ), pp. 616-627, IEEE Computer Society Press, Sept. 1999.

[5] G. Hetherington, T. Fryars, N. Tamarapalli, M. Kassab, A. Hassan, and J. Rajski, "Logic BIST for Large Industrial Designs: Real Issues and Case Studies," in Proceedings IEEE International Test Conference (ITC), (Atlantic City, NJ), pp. 358-367, IEEE Computer Society Press, Sept. 1999.

[6] Y. Zorian, S. Dey, and M. Rodgers, “Test of Future System-on-Chips,” in Proceedings International Conference on Computer-Aided Design (ICCAD), (San Jose, CA), pp. 392-398, Nov. 2000.

[7] J. Rajski, "DFT for High-Quality Low Cost Manufacturing Test," in Proceedings of the Asian Test Symposium (ATS), (Kyoto, Japan), pp. 3-8, IEEE Computer Society Press, Nov. 2001.

[8] I. Hamzaoglu and J. H. Patel, “Test set compaction algorithms for combinational circuits," in Proceedings International Conference on Computer-Aided Design (ICCAD), pp. 283-289, Nov. 1998.

[9] I. Pomeranz, L. Reddy, and S. Reddy, "COMPACTEST: A method to generate compact test set for combinational circuits," IEEE Transactions on Computer-Aided Design, vol. 12, pp. 1040-1049, July 1993. 
[10] T. Yamaguchi, M. Tilgner, M. Ishida, and D. S. Ha, "An efficient method for compressing test data to reduce the test data download time," in Proceedings IEEE International Test Conference (ITC), pp. 79-88, IEEE Computer Society Press, 1997.

[11] M. Ishida, D. S. Ha, and T. Yamaguchi, "Compact: A hybrid method for compressing tets data," in Proceedings IEEE VLSI Test Symposium (VTS), pp. 62-69, IEEE Computer Society Press, Apr. 1998.

[12] B. Koenemann, "LFSR-Coded Test Patterns for Scan Designs," in Proceedings IEEE European Test Conference (ETC), pp. 237-242, IEEE Computer Society Press, Mar. 1991.

[13] H.-J. Wunderlich and G. Kiefer, "Bit-Flipping BIST," in Proceedings International Conference on Computer-Aided Design (ICCAD), (San Jose, CA), Nov. 1996.

[14] N. A. Touba and E. J. McCluskey, "Altering a Pseudorandom Bit Sequence for Scan-Based BIST," in Proceedings IEEE International Test Conference (ITC), (Washington, DC), pp. 167-175, IEEE Computer Society Press, Oct. 1996.

[15] G. Kiefer and H.-J. Wunderlich, "Deterministic BIST with Multiple Scan Chains," in Proceedings IEEE International Test Conference (ITC), (Washington, DC), pp. 1057-1064, IEEE Computer Society Press, Oct. 1998.

[16] J. Rajski, J. Tyszer, and N. Zacharia, “Test data decompression for multiple scan designs with boundary scan," IEEE Transactions on Computers, vol. 47, pp. 1188-1200, Nov. 1998.

[17] G. Kiefer, H. Vranken, E. J. Marinissen, and H.-J. Wunderlich, “Application of Deterministic Logic BIST on Industrial Circuits," in Proceedings IEEE International Test Conference (ITC), (Atlantic City, NJ), pp. 105-114, IEEE Computer Society Press, Oct. 2000.

[18] M. L. Bushnell and V. D. Agrawal, Esentials of Electronic Testing for Digital, Memory and MixedSignal VLSI Circuits. Kluwer Academic OPTpublishers, 2000.

[19] J.-F. Li, R.-S. Tzeng, and C.-W. Wu, "Diagnostic data compression techniques for embedded memories with built-in self-test," Journal of Electronic Testing: Theory and Applications, vol. 18, pp. 515-527, Aug. 2002. 
[20] J. T. Chen, , J. Rajski, J. Khare, O. Kebichi, and W. Maly, "Enabling embedded memory diagnosis via test response compression," in Proceedings IEEE VLSI Test Symposium (VTS), (Marina del Rey, CA), pp. 292-298, IEEE Computer Society Press, May 2001.

[21] D. Das and N. A. Touba, "Reducing Test Data Volume Using External/LBIST Hybrid Test Patterns," in Proceedings IEEE International Test Conference (ITC), (Atlantic City, NJ), pp. 115-122, IEEE Computer Society Press, Oct. 2000.

[22] C. Krishna, A. Jas, and N. A. Touba, "Test Vector Encoding Using Partial LFSR Reseeding," in Proceedings IEEE International Test Conference (ITC), (Baltimore, MD), pp. 885-893, IEEE Computer Society Press, Oct. 2001.

[23] A. Jas, C. Krishna, and N. A. Touba, "Hybrid BIST Based on Weighted Pseudo-Random Testing: A New Test Resource Partitioning Scheme," in Proceedings IEEE VLSI Test Symposium (VTS), (Marina del Rey, CA), pp. 2-8, IEEE Computer Society Press, May 2001.

[24] D. Kay and S. Mourad, "Compression technique for interactive BIST application," in Proceedings IEEE VLSI Test Symposium (VTS), (Marina del Rey, CA), pp. 9-14, IEEE Computer Society Press, May 2001.

[25] D. Kay, S. Chung, and S. Mourad, "Embedded Test Control Schemes for Compression in SOCs," in Proceedings ACM/IEEE Design Automation Conference (DAC), (New Orleans, Louisiana), pp. 679-684, IEEE Computer Society Press, June 2002.

[26] R. Dorsch and H.-J. Wunderlich, “Tailoring ATPG for Embedded Testing," in Proceedings IEEE International Test Conference (ITC), (Baltimore, MD), pp. 530-537, IEEE Computer Society Press, Oct. 2001.

[27] I. Bayraktaroglu and A. Orailoglu, "Test Volume and Application Time Reduction Through Scan Chain Concealment," in Proceedings ACM/IEEE Design Automation Conference (DAC), vol. 38, pp. 151-155, June 2001. 
[28] B. Koenemann, C. Barnhart, B. Keller, T. Snethen, O. Farnsworth, and D. Wheater, "A SmartBIST variant with Guaranteed Encoding," in Proceedings of the Asian Test Symposium (ATS), (Kyoto, Japan), pp. 325-330, IEEE Computer Society Press, Nov. 2001.

[29] V. Iyengar, K. Chakrabarty, and B. Murray, "Deterministic built-in pattern generation for sequential circuits," Journal of Electronic Testing: Theory and Applications, vol. 15, pp. 97-114, August/October 1999.

[30] A. Jas and N. Touba, “Test Vector Decompression Via Cyclical Scan Chains and Its Application to Testing Core-Based Designs," in Proceedings IEEE International Test Conference (ITC), (Washington, DC), pp. 458-464, IEEE Computer Society Press, Oct. 1998.

[31] A. Jas and N. Touba, "Using an Embedded Processor for Efficient Deterministic Testing of Systems-on-a-Chip," in Proceedings International Conference on Computer Design (ICCD), (Austin, TX), pp. 418-423, Oct. 1999.

[32] A. El-Maleh, S. al Zahir, and E. Khan, "A Geometric-Primitives-Based Compression Scheme for Testing Systems-on-Chip," in Proceedings IEEE VLSI Test Symposium (VTS), pp. 114-121, IEEE Computer Society Press, Apr. 2001.

[33] S. Reda and A. Orailoglu, "Reducing Test Application Time Through Test Data Mutation Encoding," in Proceedings Design, Automation, and Test in Europe (DATE), (Paris), pp. 387-393, IEEE Computer Society Press, Mar. 2002.

[34] T. Cover and J. Thomas, Elements of Information Theory. New York: John Wiley \& Sons, 1991.

[35] V. D. Agrawal and T. J. Chakraborty, "High-Performance Circuit Testing with Slow-Speed Testers," in Proceedings IEEE International Test Conference (ITC), (Montreal, Canada), pp. 302-310, IEEE Computer Society Press, Apr. 1995.

[36] D. Heidel, S. Dhong, P. Hofstee, M. Immediato, K. Nowka, J. Silberman, and K. Stawiasz, "Highspeed serialiazing/deserializing design-for-test methods for evaluating a $1 \mathrm{ghz}$ microprocessor," in Proceedings IEEE VLSI Test Symposium (VTS), pp. 234-238, IEEE Computer Society Press, Apr. 1998. 
[37] H. Ichihara, A. Ogava, T. Inoue, and A. Tamura, "Dynamic Test Compression Using Statistical Coding," in Proceedings of the Asian Test Symposium (ATS), (Kyoto, Japan), pp. 143-148, IEEE Computer Society Press, Nov. 2001.

[38] H. Ichihara, K. Kinoshita, I. Pomeranz, and S. Reddy, "Test Transformation to Improve Compaction by Statistical Encoding," in Proceedings International Conference on VLSI Design, pp. 294-299, IEEE Computer Society Press, Jan. 2000.

[39] The University of Illinois. www.crhc.uiuc.edu/IGATE.

[40] F. Brglez, D. Bryan, and K. Kozminski, "Combinational profiles of sequential benchmark circuits," in Proceedings International Symposium on Circuits and Systems (ISCAS), pp. 1929-1934, May 1989.

[41] Synopsys Inc., "Design compiler reference manual,” 2002. 